\title{
Synthesis of DEFG ring system of cneorins
}

\author{
Ari M. P. Koskinen* and Ainoliisa J. Pihko \\ Laboratory of Organic Chemistry, Department of Chemistry, Helsinki University of Technology, \\ PO Box 6100, FI-02015 TKK, Finland \\ E-mail: ari.koskinen@tkk.fi
}

\begin{abstract}
The cneorins have been isolated from the xerophytic shrub Cneorum pulverulentum, which is native to the Canary Islands. They are natural products containing a [4.3.1]propellane ring system (DEFG rings) as the northern part of the molecule and a 5,5-spiroketal unit and a butenolide moiety (A ring) as the southern part. The synthesis of the DEFG ring system of the cneorins is described. The key steps include: intramolecular cyclopropanation of a diazomalonate providing the EFG ring fragment and an anionic cyclization of a sulfone yielding the [4.3.1]propellane ring system.
\end{abstract}

Keywords: Total synthesis, natural products, cneorins, intramolecular cyclopropanation of diazomalonates

\section{Introduction}

The cneorins (1, 2) (Figure 1) were originally isolated from the xerophytic shrub Cneorum pulverulentum, native to the Canary Islands, in the late 1970's. ${ }^{1}$ This shrub hosts a variety of bitter principles, all of which contain the [4.3.1]propellane ring system. ${ }^{2}$ These oxidized pentanortriterpenes $\left(\mathrm{C}_{25}\right.$ compounds) also have other interesting structural features in common, such as a 5,5-spiroketal unit and a butenolide moiety. The relative stereochemistry of the parent natural products were initially reported based on degradation studies, and later confirmed by Xray diffraction structures. The absolute stereochemistry has been indirectly determined for only one derivative of cneorin $\mathrm{C}$, and must be considered with caution for the other structural members of these natural products. Biogenetically these compounds are thought to be related to the limonoid triterpenes. ${ }^{3}$ Due to lack of material from natural sources, these compounds have not received proper pharmacological screening. In addition to Cneorum pulverulentum, the Cneoraceae plant family consists of two other species, Cneorum tricoccon, which is native to coastal areas of the western Mediterranean, and Cneorum trimerum, which belongs to the flora of 
Cuba. The tricoccins (3, Figure 1) were isolated from the former, but the latter has not been adequately studied due to lack of access to the required plant materials. Recently, some close structural relatives of the cneorins, the cedkathryns (4), were isolated from Cedrelopsis gracilis from Madagascar (Figure 1). ${ }^{4}$ In addition, some compounds that could result from rearrangements of the cneorins or the tricoccins, for example cedmilinol (5), have been isolated from Cedrelopsis grevei. ${ }^{5}$
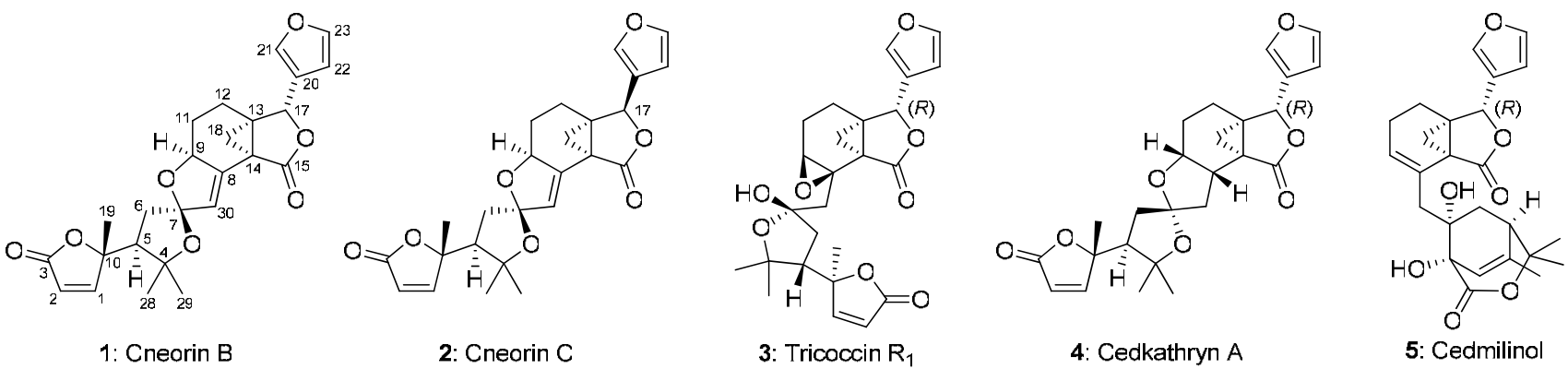

Figure 1. Selected structures of natural products related to cneorins.

Despite the intriguing structures of the cneorins, these molecules have not attracted synthetic attention except from our group. ${ }^{6}$ Retrosynthetically (Scheme 1), the structure of cneorin C can be divided into two advanced substructures, namely hydroxyketone 6, which consists of the DEFG rings of the molecule, and the A ring butenolide fragment 7 . The DEFG ring system of cneorin B (6) is an ideal candidate for the intramolecular cyclopropanation ${ }^{7-11}$ of diazomalonate 8, which can be conveniently prepared from the furyl substituted allylic alcohol (S)-9.

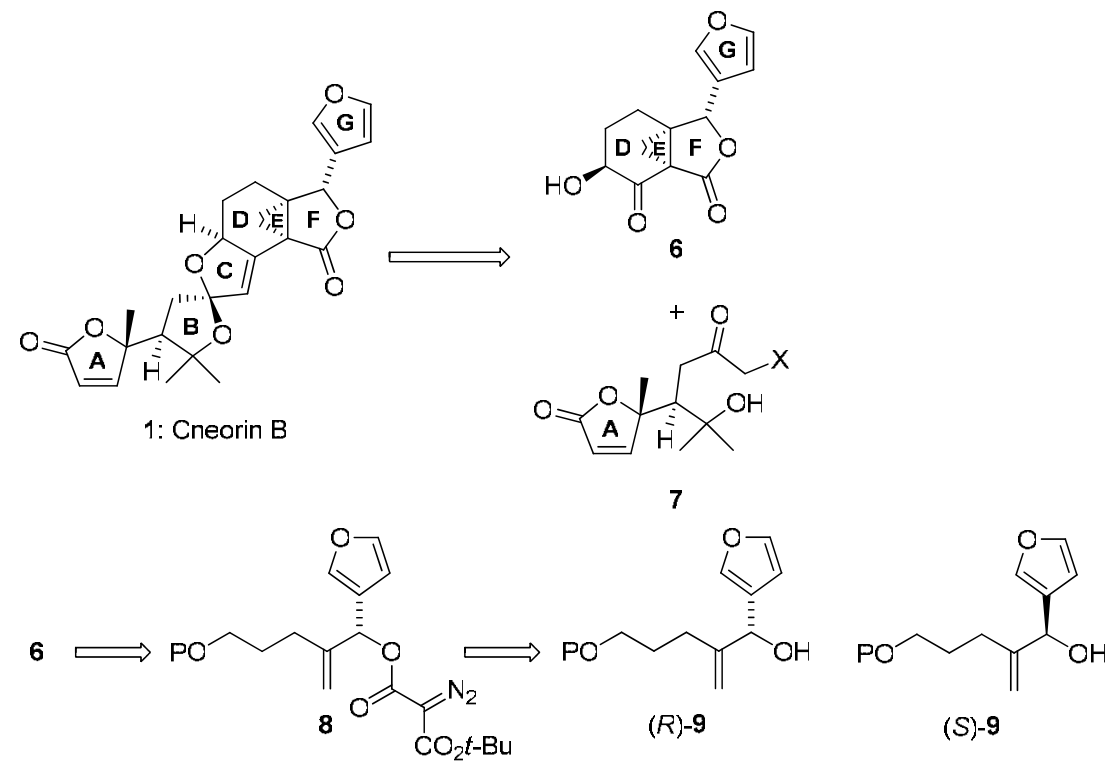

Scheme 1. Retrosynthetic analysis of cneorin B. 
We have previously described the enantioselective synthesis of allylic alcohol (S)-9 by enzymatic kinetic resolution. ${ }^{6}$ In this paper we disclose our results on the cyclopropanation to yield the EFG ring fragment and closing the D ring to obtain the [4.3.1]propellane structure of cneorin related natural products.

\section{Results and Discussion}

Transition metal catalyzed intramolecular cyclopropanation reactions have been reviewed. ${ }^{10}$ Some mechanistic studies of the intermolecular copper(I)-catalyzed cyclopropanations have also been published. ${ }^{12-14}$ The intramolecular cyclopropanation of diazomalonates of type $\mathbf{A}$ in Scheme 2 (a 1,1-disubstituted olefin) is unprecedented in the literature, although there are earlier examples of both 1,2-disubstituted and even 1,2,2-trisubstituted olefins being cyclopropanated with copper catalysts. ${ }^{8}$

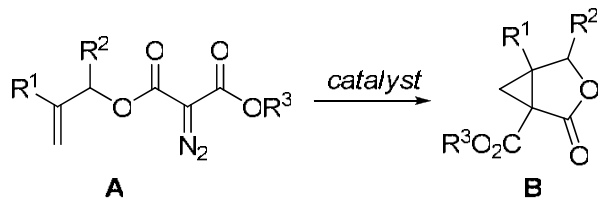

Scheme 2. Intramolecular cyclopropanation of a diazomalonate.

The catalyst complexes (Figure 2) and reaction conditions used in this study were chosen in accordance with earlier results, ${ }^{9-11}$ according to which the best results were obtained with catalyst complex 10c. ${ }^{15}$ The air stability and ease of handling of $\mathrm{Cu}(\mathrm{MeCN})_{4} \mathrm{PF}_{6}{ }^{16}$ was reported to make the $\mathrm{PF}_{6}$ salts superior to CuOTf. ${ }^{17}$ We therefore decided to investigate also complexes 10a and $\mathbf{1 0 b} \mathbf{b}^{18}$ the latter of which was expected to exhibit reduced Lewis acidity of copper thus making it less reactive and more selective towards the targeted double bond.
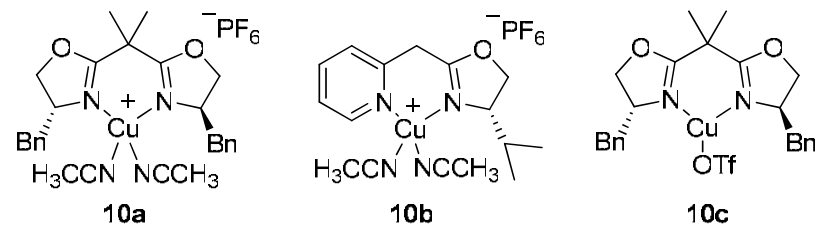

Figure 2. Catalysts employed in the intramolecular cyclopropanation.

The diazomalonates for the cyclopropanation reaction were synthesized as follows (Scheme 3 and Table 1). Alcohols 11-14 were treated with the monoester of malonic acid 15a-b in the presence of dicyclohexylcarbodiimide and $\mathrm{DMAP}^{19}$ to provide the corresponding malonates 16- 
21. Diazo transfer ${ }^{20}$ of the malonates with tosyl azide ${ }^{21}$ gave the cyclopropanation precursors 2227.

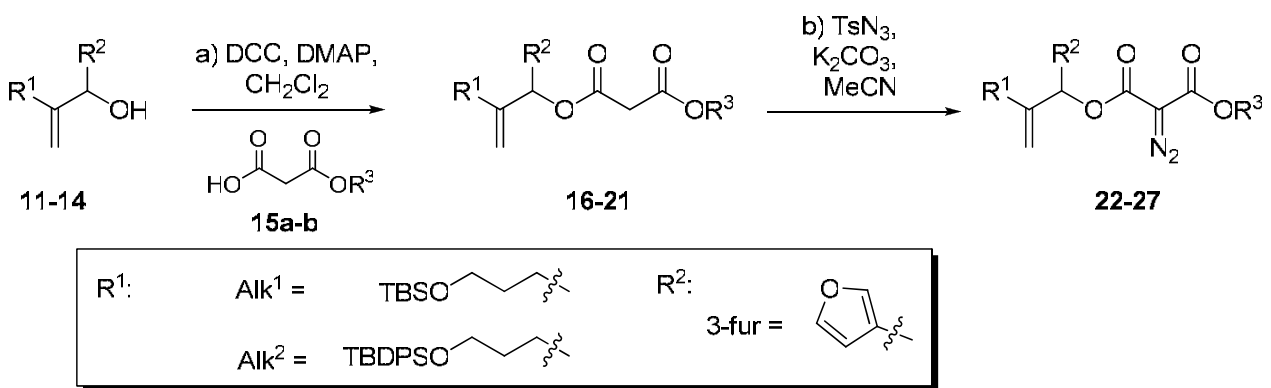

Scheme 3. Synthesis of diazomalonates. For substrates and yields, see Table 1.

Table 1. Substrates and yields for preparation of the diazomalonates (see Scheme 3)

\begin{tabular}{|c|c|c|c|c|c|c|c|c|c|}
\hline & & & & Malonic & & & Yield & Diazo- & Yield \\
\hline Entry & Alcohol & $\mathrm{R}^{1}$ & $\mathrm{R}^{2}$ & acid & $\mathrm{R}^{3}$ & Malonate & step a & malonate & step b \\
\hline 1 & 11 & $\mathrm{Me}$ & $\mathrm{Ph}$ & $15 a$ & $\mathrm{Et}$ & 16 & $63 \%$ & 22 & quant. $^{a}$ \\
\hline 2 & 12 & $\mathrm{Me}$ & 3-fur & $15 a$ & $\mathrm{Et}$ & 17 & $67 \%$ & 23 & $71 \%$ \\
\hline 3 & 13 & Alk ${ }^{1}$ & 3-fur & $15 a$ & $\mathrm{Et}$ & 18 & $81 \%$ & 24 & $92 \%$ \\
\hline 4 & 13 & $\mathrm{Alk}^{1}$ & 3-fur & $15 b$ & $t-\mathrm{Bu}$ & 19 & $88 \%$ & 25 & $93 \%$ \\
\hline 5 & 14 & $\mathrm{Alk}^{2}$ & 3-fur & $15 a$ & $\mathrm{Et}$ & 20 & $97 \%$ & 26 & quant. $^{a}$ \\
\hline 6 & 14 & $\mathrm{Alk}^{2}$ & 3-fur & $15 b$ & $t-\mathrm{Bu}$ & 21 & $98 \%{ }^{\mathrm{a}}$ & 27 & $98 \%$ \\
\hline
\end{tabular}

${ }^{a}$ Crude yield.

The ability of the furan ring to participate in carbenoid insertion reactions is well known, ${ }^{22,23}$ and indeed, the model studies confirmed this. Whereas in a model study, the phenyl substituted diazomalonate 22 upon treatment with catalyst 10c produced the corresponding cyclopropanolactone in $67 \%$ yield, the 3-furyl substituted diazomalonates were extensively decomposed with this catalyst. We soon learned that for the alcohol protection, TBS was too labile, and the ethyl allyl malonates also led to extensive decomposition. Finally, of the examined $\mathrm{Cu}$ catalyst complexes, 10a gave reproducible cyclopropanation of $\mathbf{2 7}$ to give $\mathbf{2 8}$ in $30 \%$ yield as a separable 1:1 mixture of diastereomers.

Thus, we embarked on the synthesis of both the anti-and syn-diastereomers of ketosulfone 32 with the $t$-butyl ester. The TBDPS protected anti-cyclopropanolactone 28a was deprotected with tetrabutylammonium fluoride ${ }^{24}$ to yield alcohol 29a in modest yield (Scheme 4), which was then transformed into sulfide 30a under the Hata conditions ${ }^{25,26}$ and the sulfide was oxidized with $m$-CPBA to sulfone 31a in excellent yield. Exposure of sulfone 31a to $n$-BuLi in THF at $100{ }^{\circ} \mathrm{C}$ gave the [4.3.1] propellane 32a in 55\% yield as a 1:0.8 mixture of two diastereomers at the sulfur bearing carbon. 

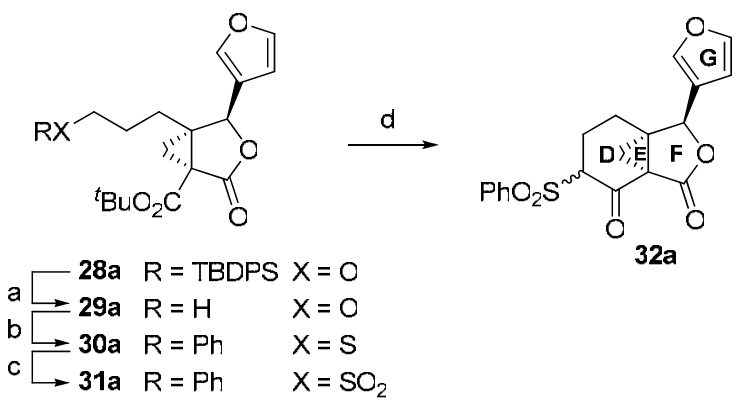

Scheme 4. (a) TBAF, THF, $0{ }^{\circ} \mathrm{C} \rightarrow \mathrm{rt}, 2 \mathrm{~h}, 57 \%$; (b) $(\mathrm{SPh})_{2}, \mathrm{Bu}_{3} \mathrm{P}, \mathrm{pyr}, 0{ }^{\circ} \mathrm{C} \rightarrow \mathrm{rt}, 1.5 \mathrm{~h}, 75 \%$; (c) $m$-CPBA, $\mathrm{NaHCO}_{3}, \mathrm{CH}_{2} \mathrm{Cl}_{2}, 0{ }^{\circ} \mathrm{C}, 1 \mathrm{~h}, 88 \%$; (d) $n$-BuLi, THF, $-100{ }^{\circ} \mathrm{C}, 30 \mathrm{~min}, 55 \%$.

In the diastereomeric series towards cneorin B and the other natural products shown in Figure 1 , the synthesis proceeded similarly, except for the closure of ring D. With the syn-phenylsulfone 31b, $n$-BuLi did not only affect the $\mathrm{D}$ ring cyclization but the $n$-butyl anion attacked the lactone as well. Fortunately, subjecting sulfone $\mathbf{3 1 b}$ to KHMDS provided the cyclized product.

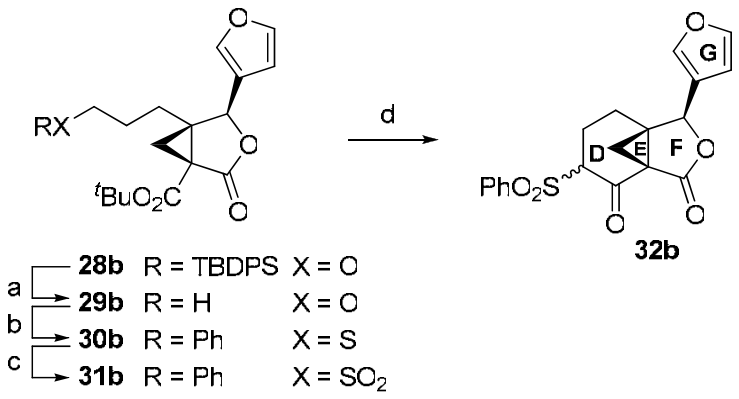

Scheme 5. (a) TBAF, THF, $0{ }^{\circ} \mathrm{C} \rightarrow \mathrm{rt}, 3 \mathrm{~h}, 80 \%$; (b) (SPh) $)_{2}, \mathrm{Bu}_{3} \mathrm{P}$, pyr, $0{ }^{\circ} \mathrm{C} \rightarrow \mathrm{rt}, 2.5 \mathrm{~h}, 65 \%$; (c) $m$-CPBA, $\mathrm{NaHCO}_{3}, \mathrm{CH}_{2} \mathrm{Cl}_{2}, 0{ }^{\circ} \mathrm{C}, 2 \mathrm{~h}, 80 \%$; (d) KHMDS, THF, $-100{ }^{\circ} \mathrm{C}, 1 \mathrm{~h}, 36 \%$ based on recovered 31b.

Having secured access to the DEFG ring system, we considered different options for removing the sulfone from 32. Single electron reductants such as Raney $\mathrm{Ni}, \mathrm{Na} / \mathrm{Hg}$ and $\mathrm{Al} / \mathrm{Hg}$ have traditionally been used to reduce the sulfur - carbon bond. Reductive opening of cyclopropyl ring systems conjugated to carbonyl groups with single electron reductants is also known in the literature. ${ }^{27-30}$

Results of the sulfone removal of the anti-diastereomer are presented in Scheme 6. Raney nickel caused slow decomposition of the starting sulfone 32a and the other single electron reductants, aluminum amalgam ${ }^{31}$ and sodium amalgam, ${ }^{32}$ effected a reductive opening of the cyclopropyl ring and ketone $\mathbf{3 3}$ was obtained in addition to decomposed starting material. ${ }^{33}$ 


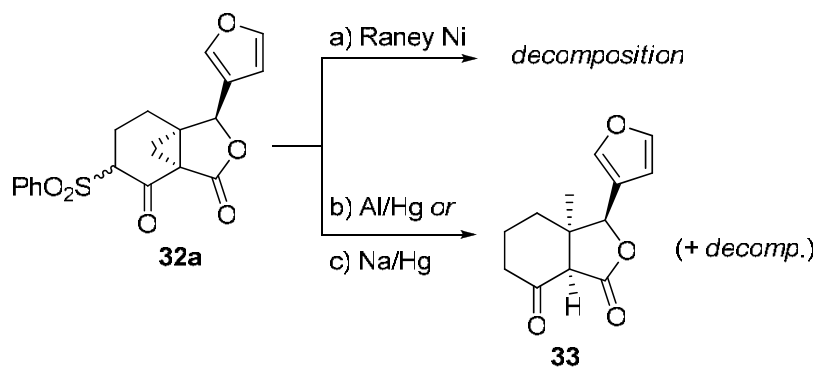

Scheme 6. (a) Raney Ni, EtOH, THF, rt, $18 \mathrm{~h}$; (b) Al-foil immersed in aq. $\mathrm{HgCl}_{2},{ }^{33} \mathrm{THF}: \mathrm{H}_{2} \mathrm{O}$ 9:1, $0{ }^{\circ} \mathrm{C} \rightarrow \mathrm{rt}$ (slowly), $4 \mathrm{~h}, 45 \%$; (c) $\mathrm{Na}-\mathrm{Hg}, \mathrm{Na}_{2} \mathrm{HPO}_{4}$, THF, $\mathrm{MeOH},-15^{\circ} \mathrm{C} \rightarrow \mathrm{rt}$ (slowly), $4 \mathrm{~h}$, yield not determined.

Undeterred by the results with the anti-diastereomer 32a, we submitted the syn-diastereomer 32b to the aluminum amalgam reduction, which to our delight produced ketone 34 (Scheme 7).

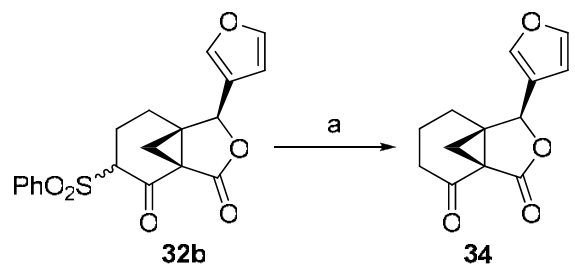

Scheme 7. (a) Al-foil immersed in aq. $\mathrm{HgCl}_{2},{ }^{33} \mathrm{THF}: \mathrm{H}_{2} \mathrm{O}$ 9:1, $0{ }^{\circ} \mathrm{C} \rightarrow \mathrm{rt}, 3 \mathrm{~h}, 40 \%$.

\section{Summary}

In this work, the synthetic efforts towards the DEFG ring system of cneorin B and C have been reported. Synthesis of cneorin $\mathrm{C}$ was hampered by difficulties in removing the sulfone from antiketosulfone 32a. Fortunately, this step was successful for the syn-diastereomer 32b.

The enantiomer of the DEFG ring fragment of cneorin B 34 was synthesized for the first time in 14 steps from commercially available starting materials. This is the first time that a cyclopropane containing [n.n.1]propellane structure of a natural product has been prepared by means of a cyclopropanation reaction.

\section{Experimental Section}

1-Furan-3-yl-2-methylprop-2-en-1-ol (12). ${ }^{34}$ To a stirred solution of 3-bromofuran $(0.90 \mathrm{~mL}$, $10.0 \mathrm{mmol}, 200 \mathrm{~mol} \%)$ in THF $(10 \mathrm{~mL})$ at $-78{ }^{\circ} \mathrm{C}$ was added $t$-BuLi $(12.4 \mathrm{~mL}, 20.0 \mathrm{mmol}$, $400 \mathrm{~mol} \%)$ dropwise. After $15 \mathrm{~min}, 2$-methyl propenal $(0.41 \mathrm{~mL}, 5.0 \mathrm{mmol}, 100 \mathrm{~mol} \%)$ was added dropwise. After $1 \mathrm{~h} 15 \mathrm{~min}$, sat. aq. $\mathrm{NH}_{4} \mathrm{Cl}(5 \mathrm{~mL})$ was added and the biphasic solution 
was allowed to warm to rt. The phases were separated, the aqueous phase was extracted with EtOAc $(3 \times 10 \mathrm{~mL})$ and the organic extracts were washed with brine, dried, filtered and concentrated. Crude alcohol $12(0.90 \mathrm{~g})$ was used without further purification for the next reaction. TLC (25\% EtOAc in hexanes): $\mathrm{R}_{\mathrm{f}}=0.3 ;{ }^{1} \mathrm{H}$ NMR: . $\quad 7.42-7.40(\mathrm{~m}, 1 \mathrm{H}), 7.38$ (br t, $J$ $=1.7 \mathrm{~Hz}, 1 \mathrm{H}), 6.34$ (br dd, $J=0.6,1.7 \mathrm{~Hz}, 1 \mathrm{H}), 5.17-5.14(\mathrm{~m}, 1 \mathrm{H}), 5.12$ (br s, 1H), 4.96-4.93 (m, $1 \mathrm{H}), 1.70$ (s, 3H).

5-(tert-Butyldimethylsilanyloxy)-1-furan-3-yl-2-methylenepentan-1-ol (13). To a stirred solution of tert-butyldimethylsilanyloxy-4-bromopent-4-ene ${ }^{35}(0.21 \mathrm{~g}, 0.76 \mathrm{mmol}, 100 \mathrm{~mol} \%)$ in THF $(4.0 \mathrm{~mL})$ at $-78{ }^{\circ} \mathrm{C}$ was added $t$-BuLi $(1.35 \mathrm{~mL}, 1.9 \mathrm{mmol}, 250 \mathrm{~mol} \%)$ dropwise. After 20 min, 3-furaldehyde (50) (95 L, $1.1 \mathrm{mmol}, 150 \mathrm{~mol} \%$ ) was added. After $40 \mathrm{~min}$, the solution was allowed to warm to $\mathrm{rt}$ and concentrated. The residue was dissolved in EtOAc $(10 \mathrm{~mL})$ and washed with sat. aq. $\mathrm{NH}_{4} \mathrm{Cl}(5 \mathrm{~mL})$. The aqueous phase was extracted with EtOAc $(2 \times 10 \mathrm{~mL})$, washed with brine, dried, filtered and concentrated. Flash column chromatography (silica, 5-15\% EtOAc in hexanes) provided furyl alcohol 13 as a colorless oil $(0.137 \mathrm{~g}, 61 \%)$. TLC (20\% EtOAc in hexanes): $\mathrm{R}_{\mathrm{f}}=0.3 ;{ }^{1} \mathrm{H}$ NMR: $\quad=7.42-7.39(\mathrm{~m}, 1 \mathrm{H}), 7.37$ (br t, $\left.J=1.7 \mathrm{~Hz}, 1 \mathrm{H}\right), 6.32$ (br dd, $J=0.7,1.7 \mathrm{~Hz}, 1 \mathrm{H}$ ), 5.22 (br s, 1H), 5.15 (br d, $J=3.7 \mathrm{~Hz}, 1 \mathrm{H}$ ), 4.97 (br d, $J=0.7 \mathrm{~Hz}$, $1 \mathrm{H}), 3.62(\mathrm{t}, J=6.2 \mathrm{~Hz}, 2 \mathrm{H}), 2.22(\mathrm{~d}, J=3.7 \mathrm{~Hz}, 1 \mathrm{H}), 2.16-1.96(\mathrm{~m}, 2 \mathrm{H}), 1.77-1.62(\mathrm{~m}, 2 \mathrm{H})$, 0.88 (s, 9H), $0.03(\mathrm{~s}, 6 \mathrm{H}) ;{ }^{13} \mathrm{C}$ NMR: $\delta=150.3,143.2,139.8,127.4,110.4,109.0,70.7,62.7$, 31.1, 28.0, 25.9, 18.3, -5.3; IR (film): $v_{\max }=3401,1650,1503 \mathrm{~cm}^{-1}$; HRMS (ESI+): $\mathrm{m} / \mathrm{z}$ calcd. for $\mathrm{C}_{16} \mathrm{H}_{28} \mathrm{O}_{3} \mathrm{NaSi} 319.1705\left(\mathrm{M}+\mathrm{Na}^{+}\right)$; found: 319.1683 .

\section{General procedure A for preparation of malonates ${ }^{19}$}

To a stirred solution of the alcohol $(100 \mathrm{~mol} \%)$, mono-ethyl malonate $(\mathbf{1 5 a})^{36}$ or mono-t-butyl malonate (15b) $)^{8,36,37}(110 \mathrm{~mol} \%)$ and DMAP $(10 \mathrm{~mol} \%)$ in $\mathrm{CH}_{2} \mathrm{Cl}_{2}(0.5 \mathrm{M})$ at $0{ }^{\circ} \mathrm{C}$ was added DCC (120 mol\%). After 1-2 h at rt, the mixture was filtered, washed twice with $\mathrm{HCl}(0.5 \mathrm{M})$ and sat. aq. $\mathrm{NaHCO}_{3}$ and once with brine, dried, filtered and concentrated.

Ethyl-2-methyl-1-phenylallyl malonate (16). Prepared according to the general procedure A with alcohol 11 (1.00 g, $6.8 \mathrm{mmol}, 100 \mathrm{~mol} \%)$, mono-ethyl malonate 15a (1.04 mL, $8.8 \mathrm{mmol}$, $130 \mathrm{~mol} \%$ ), DMAP (82 mg, $0.68 \mathrm{mmol}, 10 \mathrm{~mol} \%$ ), $\mathrm{CH}_{2} \mathrm{Cl}_{2}(35 \mathrm{~mL})$ and DCC (1.81 g, $8.8 \mathrm{mmol}$, $130 \mathrm{~mol} \%$ ). Flash column chromatography (silica, 2.5-10\% EtOAc in hexanes) provided malonate 16 as a yellowish oil $(1.12 \mathrm{~g}, 63 \%)$. TLC (20\% EtOAc in hexanes): $\mathrm{R}_{\mathrm{f}}=0.38 ;{ }^{1} \mathrm{H}$ NMR: $\delta=7.36-7.27$ (m, 5H), 6.22 (br s, 1H), 5.14 (br d, $J=0.8 \mathrm{~Hz}, 1 \mathrm{H}), 5.01$ (br d, $J=0.8 \mathrm{~Hz}$, $1 \mathrm{H}), 4.19(\mathrm{q}, J=7.1 \mathrm{~Hz}, 2 \mathrm{H}), 3.44(\mathrm{~s}, 2 \mathrm{H}), 1.65(\mathrm{~s}, 3 \mathrm{H}), 1.25(\mathrm{t}, J=7.1 \mathrm{~Hz}, 3 \mathrm{H}) ;{ }^{13} \mathrm{C} \mathrm{NMR}: \delta=$ $166.3,165.4,142.5,137.8,128.4,128.2,127.0,113.1,79.5,61.5,41.9,18.7,14.0$; IR (film): $v_{\max }=1752,1736,1654 \mathrm{~cm}^{-1}$; HRMS (ESI+): $\mathrm{m} / \mathrm{z}$ calcd. for $\mathrm{C}_{15} \mathrm{H}_{18} \mathrm{O}_{4} \mathrm{Na} 285.1103\left(\mathrm{M}+\mathrm{Na}^{+}\right)$; found: 285.1118 .

Ethyl-1-furan-3-yl-2-methylallyl malonate (17). Prepared according to the general procedure A with alcohol 12 (crude, $0.69 \mathrm{~g}, 5.0 \mathrm{mmol}, 100 \mathrm{~mol} \%$ ), mono-ethyl malonate 15a $(0.77 \mathrm{~mL}$, $6.5 \mathrm{mmol}, 130 \mathrm{~mol} \%)$, DMAP (61 mg, $0.50 \mathrm{mmol}, 10 \mathrm{~mol} \%), \mathrm{CH}_{2} \mathrm{Cl}_{2}(25 \mathrm{~mL})$ and DCC (1.34 g, $6.5 \mathrm{mmol}, 130 \mathrm{~mol} \%$ ). Flash column chromatography (silica, 2.5-7.5 \% EtOAc in hexanes) 
provided the malonate 17 as a colorless oil $(0.84 \mathrm{~g}, 67 \%)$. TLC (20\% EtOAc in hexanes): $\mathrm{R}_{\mathrm{f}}=$ 0.4; ${ }^{1} \mathrm{H}$ NMR: $\delta=7.44-7.41(\mathrm{~m}, 1 \mathrm{H}), 7.37$ (br t, $\left.J=1.7 \mathrm{~Hz}, 1 \mathrm{H}\right), 6.33$ (br dd, $J=0.6,1.7 \mathrm{~Hz}$, 1H), 6.20 (br s, 1H), 5.13 (br d, $J=0.8 \mathrm{~Hz}, 1 \mathrm{H}$ ), 5.00 (br d, $J=0.8 \mathrm{~Hz}, 1 \mathrm{H}$ ), 4.20 (q, $J=7.2 \mathrm{~Hz}$, 2H), $3.41(\mathrm{~s}, 2 \mathrm{H}), 1.72(\mathrm{~s}, 3 \mathrm{H}), 1.26(\mathrm{t}, J=7.2 \mathrm{~Hz}, 3 \mathrm{H}) ;{ }^{13} \mathrm{C} \mathrm{NMR}: \delta=166.3,165.4,143.3$, 141.8, 140.8, 123.0, 113.2, 109.3, 72.7, 61.6, 41.8, 18.7, 14.0; IR (film): $v_{\max }=1734,1655 \mathrm{~cm}^{-1}$; HRMS (ESI+): $\mathrm{m} / \mathrm{z}$ calcd. for $\mathrm{C}_{13} \mathrm{H}_{16} \mathrm{O}_{5} \mathrm{Na} 275.0895\left(\mathrm{M}+\mathrm{Na}^{+}\right)$; found: 275.0901 .

Ethyl-5-(tert-butyldimethylsilanyloxy)-1-furan-3-yl-2-methylenepentyl malonate (18). Prepared according to the general procedure A with alcohol 13 (58 mg, $0.20 \mathrm{mmol}, 100 \mathrm{~mol} \%$ ), mono-ethyl malonate 15a (25 $\mu \mathrm{L}, 0.22 \mathrm{mmol}, 110 \mathrm{~mol} \%)$, DMAP (2.4 mg, $20 \mu \mathrm{mol}, 10 \mathrm{~mol} \%$ ), $\mathrm{CH}_{2} \mathrm{Cl}_{2}(0.4 \mathrm{~mL})$ and DCC $(61 \mathrm{mg}, 0.30 \mathrm{mmol}, 150 \mathrm{~mol} \%)$. Flash column chromatography (silica, 2.5-7.5\% EtOAc in hexanes) provided malonate 18 as a yellowish oil (65 $\mathrm{mg}, 81 \%$ ). TLC (20\% EtOAc in hexanes): $\mathrm{R}_{\mathrm{f}}=0.48 ;{ }^{1} \mathrm{H}$ NMR: $\delta=7.44-7.40(\mathrm{~m}, 1 \mathrm{H}), 7.36(\mathrm{br} \mathrm{t}, J=1.7 \mathrm{~Hz}, 1 \mathrm{H})$, $6.33($ br s, 1H), $6.22($ br s, $1 \mathrm{H}), 5.20(\mathrm{~s}, 1 \mathrm{H}), 5.02(\mathrm{~s}, 1 \mathrm{H}), 4.19(\mathrm{q}, J=7.1 \mathrm{~Hz}, 2 \mathrm{H}), 3.58(\mathrm{t}, J=$ $6.4 \mathrm{~Hz}, 2 \mathrm{H}), 3.39(\mathrm{~s}, 2 \mathrm{H}), 2.14-1.98(\mathrm{~m}, 2 \mathrm{H}), 1.71-1.62(\mathrm{~m}, 2 \mathrm{H}), 1.25(\mathrm{t}, J=7.1 \mathrm{~Hz}, 3 \mathrm{H}), 0.86(\mathrm{~s}$, 9H), $0.02(\mathrm{~s}, 6 \mathrm{H}) ;{ }^{13} \mathrm{C}$ NMR: $\delta=166.3,165.4,145.9,143.3,141.1,123.2,111.7,109.5,72.0$, $62.5,61.5,41.8,30.8,28.5,25.9,18.3,14.0,-5.3$; IR (film): $v_{\max } 1752,1735,1650,1503 \mathrm{~cm}^{-1}$; HRMS (ESI+): $m / z$ calcd. for $\mathrm{C}_{21} \mathrm{H}_{34} \mathrm{O}_{6} \mathrm{NaSi} 433.2022\left(\mathrm{M}+\mathrm{Na}^{+}\right)$; found: 433.2036.

tert-Butyl-5-(tert-butyldimethylsilanyloxy)-1-furan-3-yl-2-methylenepentyl malonate (19). Prepared according to the general procedure A with alcohol 13 (71 mg, $0.24 \mathrm{mmol}, 100 \mathrm{~mol} \%$ ), mono-t-butyl malonate 15b (26 $\mu \mathrm{L}, 0.29 \mathrm{mmol}, 120 \mathrm{~mol} \%$ ), DMAP (2.9 mg, $24 \mu \mathrm{mol}$, $10 \mathrm{~mol} \%), \mathrm{CH}_{2} \mathrm{Cl}_{2}(0.5 \mathrm{~mL})$ and DCC (74 $\left.\mathrm{mg}, 0.36 \mathrm{mmol}, 150 \mathrm{~mol} \%\right)$. Flash column chromatography (silica, 2.5-10\% EtOAc in hexanes) provided malonate 19 as a yellowish oil (92 mg, 88\%). TLC (20\% EtOAc in hexanes): $\mathrm{R}_{\mathrm{f}}=0.6 ;{ }^{1} \mathrm{H}$ NMR: $\delta=7.42$ (br s, $\left.1 \mathrm{H}\right), 7.36$ (br t, $J=1.7 \mathrm{~Hz}, 1 \mathrm{H}), 6.33($ br s, 1H), $6.21($ br s, $1 \mathrm{H}), 5.21(\mathrm{~s}, 1 \mathrm{H}), 5.02(\mathrm{~s}, 1 \mathrm{H}), 3.58(\mathrm{t}, J=6.3 \mathrm{~Hz}$, $2 \mathrm{H}), 3.31(\mathrm{~s}, 2 \mathrm{H}), 2.12-1.98(\mathrm{~m}, 2 \mathrm{H}), 1.69-1.61(\mathrm{~m}, 2 \mathrm{H}), 1.43(\mathrm{~s}, 9 \mathrm{H}), 0.86(\mathrm{~s}, 9 \mathrm{H}), 0.02(\mathrm{~s}, 6 \mathrm{H})$; ${ }^{13} \mathrm{C}$ NMR: $\delta=165.8,165.4,145.9,143.2,141.1,123.3,111.5,109.5,82.1,71.7,62.5,43.2,30.8$, 28.5, 27.9, 25.9, 18.3, -5.3; IR (film): $v_{\max }=1749,1732,1651,1503 \mathrm{~cm}^{-1}$; HRMS (ESI+) $\mathrm{m} / \mathrm{z}$ calcd. for $\mathrm{C}_{23} \mathrm{H}_{38} \mathrm{O}_{6} \mathrm{NaSi} 461.2335\left(\mathrm{M}+\mathrm{Na}^{+}\right)$; found: 461.2360 .

Ethyl-5-(tert-butyldiphenylsilanyloxy)-(1S)-furan-3-yl-2-methylenepentyl malonate (20). Prepared according to the general procedure A with alcohol $(S)-14$ (0.86 g, $2.1 \mathrm{mmol}$, $100 \mathrm{~mol} \%)$, mono-ethyl malonate 15a $(0.24 \mathrm{~mL}, 2.1 \mathrm{mmol}, 100 \mathrm{~mol} \%)$, DMAP (25 mg, $0.21 \mathrm{mmol}, 10 \mathrm{~mol} \%), \mathrm{CH}_{2} \mathrm{Cl}_{2}(4.1 \mathrm{~mL})$ and DCC (0.55 g, $\left.2.7 \mathrm{mmol}, 130 \mathrm{~mol} \%\right)$. Dry-column flash chromatography ${ }^{38}$ (silica, 0-20\% EtOAc in hexanes) provided malonate 20 as a yellowish oil (1.06 g, 97\%). TLC (20\% EtOAc in hexanes): $\mathrm{R}_{\mathrm{f}}=0.43 ;[\alpha]_{20}{ }^{\mathrm{D}}-7.9$ (c 1.0, $\mathrm{CHCl}_{3} ; 59 \%$ ee); ${ }^{1} \mathrm{H}$ NMR: $\delta=7.68-7.62(\mathrm{~m}, 4 \mathrm{H}), 7.45-7.35(\mathrm{~m}, 8 \mathrm{H}), 6.33(\mathrm{br} \mathrm{dd}, J=1.8,0.6 \mathrm{~Hz}, 1 \mathrm{H}), 6.22$ (br s, $1 \mathrm{H}), 5.21$ (br s, 1H), $5.00(\mathrm{br} \mathrm{s}, 1 \mathrm{H}), 4.19(\mathrm{q}, J=7.2 \mathrm{~Hz}, 2 \mathrm{H}), 3.66(\mathrm{t}, J=6.3 \mathrm{~Hz}, 2 \mathrm{H}), 3.40$ (s, $2 \mathrm{H}), 2.20-2.03(\mathrm{~m}, 2 \mathrm{H}), 1.77-1.65(\mathrm{~m}, 2 \mathrm{H}), 1.25(\mathrm{t}, J=7.2 \mathrm{~Hz}, 3 \mathrm{H}), 1.04(\mathrm{~s}, 9 \mathrm{H}) ;{ }^{13} \mathrm{C}$ NMR: $\delta=$ $166.3,165.4,145.8,143.3,141.0,135.5,133.9,129.5,127.6,123.1,111.6,109.4,71.9,63.2$, 61.5, 41.8, 30.5, 28.4, 26.8, 19.2, 14.0; IR (film): $v_{\max }=1735 \mathrm{~cm}^{-1}$; HRMS (ESI+): $\mathrm{m} / \mathrm{z}$ calcd. for $\mathrm{C}_{31} \mathrm{H}_{38} \mathrm{O}_{6} \mathrm{NaSi} 557.2335\left(\mathrm{M}+\mathrm{Na}^{+}\right)$; found: 557.2337 . 
tert-Butyl-5-(tert-butyldiphenylsilanyloxy)-(1S)-furan-3-yl-2-methylenepentyl

malonate

(21). Prepared according to the general procedure A with alcohol $(S)-14(0.87 \mathrm{~g}, 2.1 \mathrm{mmol}$, $100 \mathrm{~mol} \%$ ), mono-t-butyl malonate 15b (0.33 g, $2.1 \mathrm{mmol}, 100 \mathrm{~mol} \%)$, DMAP (25 mg, $0.21 \mathrm{mmol}, 10 \mathrm{~mol} \%)$, DCC (0.51 g, $2.5 \mathrm{mmol}, 120 \mathrm{~mol} \%)$ and $\mathrm{CH}_{2} \mathrm{Cl}_{2}$ (4.2 $\left.\mathrm{mL}\right)$. The reaction provided malonate 21 as a yellow oil (1.14 g, 98\%), which was sufficiently pure to be used in the next step. An analytical sample was purified by dry-column flash chromatography ${ }^{[39]}$ (silica, 0 $15 \%$ EtOAc in hexanes). TLC (20\% EtOAc in hexanes): $\mathrm{R}_{\mathrm{f}}=0.40 ;[\alpha]_{20}{ }^{\mathrm{D}}-19$ (c 1.0, CHCl3); ${ }^{1} \mathrm{H}$ NMR: $\delta=7.68-7.61(\mathrm{~m}, 4 \mathrm{H}), 7.45-7.34(\mathrm{~m}, 8 \mathrm{H}), 6.32(\mathrm{br} \mathrm{dd}, \mathrm{J}=1.7,0.6 \mathrm{~Hz}, 1 \mathrm{H}), 6.21$ (br s, 1H), 5.21 (br s, 1H), 4.99 (br s, 1H), 3.64 (t, J = 6.3 Hz, 2H), 3.30 (s, 2H), 2.18-2.02 (m, 2H), 1.76-1.64 (m, 2H), $1.43(\mathrm{~s}, 9 \mathrm{H}), 1.02(\mathrm{~s}, 9 \mathrm{H}) ;{ }^{13} \mathrm{C}$ NMR: $\delta=165.7,165.4,145.8,143.2,141.0$, 135.5, 133.9, 129.5, 127.6, 123.2, 111.5, 109.4, 82.0, 71.6, 63.2, 43.1, 30.5, 28.5, 27.8, 26.8, 19.1; IR (film): $v_{\max }=1728,1649,1588 \mathrm{~cm}^{-1}$; HRMS (ESI+): $\mathrm{m} / \mathrm{z}$ calcd. for $\mathrm{C}_{33} \mathrm{H}_{42} \mathrm{O}_{5} \mathrm{NaSi}$ 585.2648 $\left(\mathrm{M}+\mathrm{Na}^{+}\right)$; found: 585.2625 .

\section{General procedure $B$ for preparation of diazomalonates ${ }^{21}$}

To a stirred solution of the malonate $(100 \mathrm{~mol} \%)$ in $\mathrm{MeCN}(0.5 \mathrm{M})$ was added powdered and dried $\mathrm{K}_{2} \mathrm{CO}_{3}(105 \mathrm{~mol} \%)$ followed by $\mathrm{TsN}_{3}(105 \mathrm{~mol} \%)^{20}$ in $\mathrm{MeCN}$. After $2 \mathrm{~h}$, the solvent was evaporated. The residue was dissolved in $\mathrm{Et}_{2} \mathrm{O}$ and the resulting solution washed twice with $\mathrm{K}_{2} \mathrm{CO}_{3}$ (10\% aq. soln.), dried, filtered and concentrated. The residue was dissolved in $\mathrm{Et}_{2} \mathrm{O}$ and hexanes were added to precipitate $\mathrm{TsNH}_{2}$. The mixture was filtered through a pad of silica (10\% $\mathrm{Et}_{2} \mathrm{O}$ in hexanes flush) and concentrated. Generally the products were used without further purification for the cyclopropanation reaction.

Ethyl-2-methyl-1-phenylallyl diazomalonate (22). Prepared according to the general procedure B with malonate 16 (1.00 g, $3.8 \mathrm{mmol}, 100 \mathrm{~mol} \%$ ), MeCN (7.6 mL), TsN 3 (0.79 g, $4.0 \mathrm{mmol}$, $105 \mathrm{~mol} \%)$ and $\mathrm{K}_{2} \mathrm{CO}_{3}(0.55 \mathrm{~g}, 4.0 \mathrm{mmol}, 105 \mathrm{~mol} \%)$. The reaction provided diazomalonate 22 as a yellow oil $(1.2 \mathrm{~g})$, which was sufficiently pure for the next reaction. TLC (20\% EtOAc in hexanes): $\mathrm{R}_{\mathrm{f}}=0.38 ;{ }^{1} \mathrm{H}$ NMR: $\delta=7.40-7.26(\mathrm{~m}, 5 \mathrm{H}), 6.31$ (br s, $\left.1 \mathrm{H}\right), 5.19(\mathrm{br} \mathrm{d}, J=0.8 \mathrm{~Hz}, 1 \mathrm{H})$, $5.01(\mathrm{br} \mathrm{d}, J=0.8 \mathrm{~Hz}, 1 \mathrm{H}), 4.31(\mathrm{q}, J=7.1 \mathrm{~Hz}, 2 \mathrm{H}), 1.65(\mathrm{~s}, 3 \mathrm{H}), 1.32(\mathrm{t}, J=7.1 \mathrm{~Hz}, 3 \mathrm{H}) ;{ }^{13} \mathrm{C}$ NMR: $\delta=160.8,160.2,142.5,137.9,128.4,128.1,126.9,113.0,79.5,61.6,18.5,14.3$; IR (film): $v_{\max }=2142,1761,1735,1693,1653 \mathrm{~cm}^{-1}$; HRMS (ESI+): $\mathrm{m} / \mathrm{z}$ calcd. for $\mathrm{C}_{15} \mathrm{H}_{16} \mathrm{~N}_{2} \mathrm{O}_{4} \mathrm{Na}$ $311.1008\left(\mathrm{M}+\mathrm{Na}^{+}\right)$; found: 311.1003 .

Ethyl-1-furan-3-yl-2-methylallyl diazomalonate (23). Prepared according to the general procedure B with malonate 17 (0.82 g, $3.2 \mathrm{mmol}, 100 \mathrm{~mol} \%), \mathrm{MeCN}(6.5 \mathrm{~mL}), \mathrm{TsN}_{3}(0.67 \mathrm{~g}$, $3.4 \mathrm{mmol}, 105 \mathrm{~mol} \%)$ and $\mathrm{K}_{2} \mathrm{CO}_{3}(0.47 \mathrm{~g}, 3.4 \mathrm{mmol}, 105 \mathrm{~mol} \%)$. The reaction provided diazomalonate 23 as a yellow oil $(0.64 \mathrm{~g}, 71 \%)$. TLC (20\% EtOAc in hexanes): $\mathrm{R}_{\mathrm{f}}=0.4 ;{ }^{1} \mathrm{H}$ NMR: $\delta=7.46-7.44(\mathrm{~m}, 1 \mathrm{H}), 7.37$ (br t, $J=1.7 \mathrm{~Hz}, 1 \mathrm{H}), 6.35$ (br dd, $J=1.7,0.7 \mathrm{~Hz}, 1 \mathrm{H}), 6.28$ (br s, 1H), 5.16 (br d, $J=0.8 \mathrm{~Hz}, 1 \mathrm{H}), 5.01$ (br d, $J=0.8 \mathrm{~Hz}, 1 \mathrm{H}), 4.30$ (q, $J=7.1 \mathrm{~Hz}, 2 \mathrm{H}), 1.72$ $(\mathrm{s}, 3 \mathrm{H}), 1.31(\mathrm{t}, J=7.1 \mathrm{~Hz}, 3 \mathrm{H}) ;{ }^{13} \mathrm{C}$ NMR: $\delta=160.8,160.3,143.3,141.9,141.0,123.1,113.1$, 109.2, 72.6, 61.7, 18.6, 14.3; IR (film): $v_{\max }=2142,1759,1732,1690,1655,1504 \mathrm{~cm}^{-1}$; HRMS (ESI+): $\mathrm{m} / \mathrm{z}$ calcd. for $\mathrm{C}_{13} \mathrm{H}_{14} \mathrm{~N}_{2} \mathrm{O}_{5} \mathrm{Na} 301.0800\left(\mathrm{M}+\mathrm{Na}^{+}\right)$; found: 301.0817 . 
Ethyl-5-(tert-butyldimethylsilanyloxy)-1-furan-3-yl-2-methylenepentyl diazomalonate (24). Prepared according to the general procedure B with malonate 18 (60 mg, $0.15 \mathrm{mmol}, 100 \mathrm{~mol} \%$ ), $\mathrm{MeCN}(0.3 \mathrm{~mL}), \mathrm{TsN}_{3}(30 \mathrm{mg}, 0.15 \mathrm{mmol}, 105 \mathrm{~mol} \%)$ and $\mathrm{K}_{2} \mathrm{CO}_{3}(21 \mathrm{mg}, 0.15 \mathrm{mmol}$, $105 \mathrm{~mol} \%)$. The reaction provided diazomalonate 24 as a yellow oil (59 $\mathrm{mg}, 92 \%)$. TLC (20\% EtOAc in hexanes): $\mathrm{R}_{\mathrm{f}}=0.48 ;{ }^{1} \mathrm{H}$ NMR: $\delta=7.46-7.43(\mathrm{~m}, 1 \mathrm{H}), 7.37$ (br t, $\left.\mathrm{J}=1.6 \mathrm{~Hz}, 1 \mathrm{H}\right), 6.36-$ $6.34(\mathrm{~m}, 1 \mathrm{H}), 6.30$ (br s, 1H), 5.24 (s, 1H), 5.03 (s, 1H), 4.30 (q, J = 7.1 Hz, 2H), 3.58 (t, J = 6.3 $\mathrm{Hz}, 2 \mathrm{H}), 2.15-1.98(\mathrm{~m}, 2 \mathrm{H}), 1.70-1.61(\mathrm{~m}, 2 \mathrm{H}), 1.31(\mathrm{t}, \mathrm{J}=7.1 \mathrm{~Hz}, 3 \mathrm{H}), 0.87(\mathrm{~s}, 9 \mathrm{H}), 0.02(\mathrm{~s}$, $6 \mathrm{H}) ;{ }^{13} \mathrm{C}$ NMR: $\delta=160.8,160.2,145.9,143.3,141.2,123.2,111.6,109.4,71.9,62.4,61.7,30.8$, 28.5, 25.9, 18.2, 14.3, -5.4; IR (film): $v_{\max }=2141,1762,1735,1693,1651,1503 \mathrm{~cm}^{-1}$; HRMS (ESI+): $m / z$ calcd. for $\mathrm{C}_{21} \mathrm{H}_{32} \mathrm{~N}_{2} \mathrm{O}_{6} \mathrm{NaSi} 459.1927\left(\mathrm{M}+\mathrm{Na}^{+}\right)$; found: 459.1930 .

tert-Butyl-5-(tert-butyldimethylsilanyloxy)-1-furan-3-yl-2-methylenepentyl diazomalonate (25). Prepared according to the general procedure B with malonate 19 (80 $\mathrm{mg}, 0.18 \mathrm{mmol}$, $100 \mathrm{~mol} \%), \mathrm{MeCN}(0.4 \mathrm{~mL}), \mathrm{TsN}_{3}(38 \mathrm{mg}, 0.19 \mathrm{mmol}, 105 \mathrm{~mol} \%)$ and $\mathrm{K}_{2} \mathrm{CO}_{3}(27 \mathrm{mg}$, $0.19 \mathrm{mmol}, 105 \mathrm{~mol} \%)$. The reaction provided diazomalonate 25 as a yellow oil (79 $\mathrm{mg}, 93 \%)$. TLC (20\% EtOAc in hexanes): $\mathrm{R}_{\mathrm{f}}=0.6 ;{ }^{1} \mathrm{H}$ NMR: $\delta=7.45-7.42(\mathrm{~m}, 1 \mathrm{H}), 7.36$ (br t, $J=1.6 \mathrm{~Hz}$, $1 \mathrm{H}), 6.36-6.33(\mathrm{~m}, 1 \mathrm{H}), 6.31(\mathrm{br} \mathrm{s}, 1 \mathrm{H}), 5.22(\mathrm{~s}, 1 \mathrm{H}), 5.03(\mathrm{~s}, 1 \mathrm{H}), 3.58(\mathrm{t}, J=6.3 \mathrm{~Hz}, 2 \mathrm{H}), 2.14-$ $1.98(\mathrm{~m}, 2 \mathrm{H}), 1.70-1.60(\mathrm{~m}, 2 \mathrm{H}), 1.51(\mathrm{~s}, 9 \mathrm{H}), 0.87(\mathrm{~s}, 9 \mathrm{H}), 0.02(\mathrm{~s}, 6 \mathrm{H}) ;{ }^{13} \mathrm{C} \mathrm{NMR}: \delta=160.4$, $159.7,146.0,143.3,141.2,123.3,111.6,109.4,83.1,71.5,62.4,30.8,28.5,28.2,25.9,18.3$, 5.4; IR (film): $v_{\max }=2137,1759,1731,1688,1503 \mathrm{~cm}^{-1}$; HRMS (ESI+): $\mathrm{m} / \mathrm{z}$ calcd. for C23H36N2O6NaSi 487.2224 $\left(\mathrm{M}+\mathrm{Na}^{+}\right)$; found: 487.2240.

Ethyl-5-(tert-butyldiphenylsilanyloxy)-(1S)-furan-3-yl-2-methylenepentyl diazomalonate (26). Prepared according to the general procedure B with malonate 20 (1.06 g, $2.0 \mathrm{mmol}$, $100 \mathrm{~mol} \%$ ), $\mathrm{MeCN}(4.0 \mathrm{~mL}), \mathrm{TsN}_{3}(0.41 \mathrm{~g}, 2.1 \mathrm{mmol}, 105 \mathrm{~mol} \%)$ and $\mathrm{K}_{2} \mathrm{CO}_{3}(0.29 \mathrm{~g}, 2.1 \mathrm{mmol}$, $105 \mathrm{~mol} \%$ ). Reaction provided diazomalonate 26 as a yellow oil (1.10 g, quant.). TLC (20\% EtOAc in hexanes): $\mathrm{R}_{\mathrm{f}}=0.43 ;[\alpha]_{20}{ }^{\mathrm{D}}-15.9$ (c 1.0, CHCl3; 59\% ee); ${ }^{1} \mathrm{H}$ NMR: $\delta=7.67-7.61(\mathrm{~m}$, 4H), 7.45-7.33 (m, 8H), 6.34 (br dd, J = 1.8, 0.6 Hz, 1H), 6.30 (br s, 1H), 5.23 (br s, 1H), 5.01 (br s, 1H), 4.30 (q, J = 7.1 Hz, 2H), $3.64(\mathrm{t}, \mathrm{J}=6.2 \mathrm{~Hz}, 2 \mathrm{H}), 2.19-2.04(\mathrm{~m}, 2 \mathrm{H}), 1.76-1.65$ (m, $2 \mathrm{H}), 1.31(\mathrm{t}, \mathrm{J}=7.1 \mathrm{~Hz}, 3 \mathrm{H}), 1.03(\mathrm{~s}, 9 \mathrm{H}) ;{ }^{13} \mathrm{C} \mathrm{NMR}: \delta=160.8,160.2,145.8,143.3,141.2$, $135.5,133.9,129.5,127.6,123.1,111.7,109.3,71.8,63.2,61.7,30.5,28.5,26.8$ 19.2, 14.3; IR (film): $v_{\max }=2141,1759,1734,1691,1589,1503 \mathrm{~cm}^{-1} ;$ HRMS (ESI+): $\mathrm{m} / \mathrm{z}$ calcd. for $\mathrm{C}_{31} \mathrm{H}_{36} \mathrm{~N}_{2} \mathrm{O}_{6} \mathrm{NaSi} 583.2240\left(\mathrm{M}+\mathrm{Na}^{+}\right)$; found:583.2226.

\section{tert-Butyl-5-(tert-butyldiphenylsilanyloxy)-(1S)-furan-3-yl-2-methylenepentyl}

diazomalonate (27). Prepared according to the general procedure B with malonate 21 (2.06 g, $3.7 \mathrm{mmol}, 100 \mathrm{~mol} \%), \mathrm{MeCN}(7.7 \mathrm{~mL}), \mathrm{K}_{2} \mathrm{CO}_{3}(0.56 \mathrm{~g}, 4.1 \mathrm{mmol}, 110 \mathrm{~mol} \%)$ and $\mathrm{TsN}_{3}(0.80 \mathrm{~g}$, $4.1 \mathrm{mmol}, 100 \mathrm{~mol} \%$ ). Dry column flash chromatography2 (silica, 5-15\% EtOAc in hexanes) provided diazomalonate 27 as a yellow oil $(2.11 \mathrm{~g}, 98 \%)$. TLC (20\% EtOAc in hexanes): $\mathrm{R}_{\mathrm{f}}=$ 0.4; $[\alpha]_{20}{ }^{\mathrm{D}}-22\left(\mathrm{c} 1.0, \mathrm{CHCl}_{3}\right)$; ${ }^{1} \mathrm{H}$ NMR: $\delta=7.68-7.61(\mathrm{~m}, 4 \mathrm{H}), 7.45-7.33(\mathrm{~m}, 8 \mathrm{H}), 6.33(\mathrm{br} \mathrm{dd}$, $\mathrm{J}=1.8,0.7 \mathrm{~Hz}, 1 \mathrm{H}), 6.30$ (br s, 1H), 5.20 (br s, 1H), 4.99 (br s, 1H), 3.64 (t, J = 6.2 Hz, 2H), 2.18-2.02 (m, 2H), 1.74-1.64 (m, 2H), $1.51(\mathrm{~s}, 9 \mathrm{H}), 1.02(\mathrm{~s}, 9 \mathrm{H}) ;{ }^{13} \mathrm{C}$ NMR: $\delta=168.6,159.6$, $145.9,143.2,141.2,135.5,133.9,129.5,127.6,123.2,111.6,109.4,83.1,71.4,63.2,30.6,28.5$, 
28.2, 26.8, 19.1; IR (film): $v_{\max }=2137,1755,1729,1686,1588 \mathrm{~cm}^{-1}$; HRMS (ESI+): $\mathrm{m} / \mathrm{z}$ calcd. for $\mathrm{C}_{33} \mathrm{H}_{40} \mathrm{~N}_{2} \mathrm{O}_{6} \mathrm{NaSi} 611.2553\left(\mathrm{M}+\mathrm{Na}^{+}\right)$; found: 611.2548 .

(5S)-[3-(tert-Butyldimethylsilanyloxy)propyl]-(4S)-furan-3-yl-2-oxo-3oxabicyclo[3.1.0]hexane-(1S)-carboxylic acid tert-butyl ester (28a) and (5R)-[3-(tertButyldimethylsilanyloxy)propyl]-(4S)-furan-3-yl-2-oxo-3-oxabicyclo[3.1.0]hexane-(1R)carboxylic acid tert-butyl ester (28b)To a stirred solution of diazomalonate 27 (1.15 g, $2.0 \mathrm{mmol}, 100 \mathrm{~mol} \%)$ in DCE $(17 \mathrm{~mL})$ was added the catalytic complex 10a $(38 \mathrm{mg}, 59 \mu \mathrm{mol}$, $3 \mathrm{~mol} \%$ ). The solution was heated up to $55^{\circ} \mathrm{C}$, the temperature at which the mixture started to effervesce and turned brown in color. After $24 \mathrm{~h}$, all diazocompound had been consumed as judged by TLC, the solution was cooled to $\mathrm{rt}$ and concentrated. The residue was dissolved in MTBE, stirred with activated carbon for $5 \mathrm{~min}$, filtered through a pad of Celite ${ }^{\circledR}$ and concentrated. Flash column chromatography (silica, 5-15\% EtOAc in hexanes) provided cyclopropanolactones 28a and 28b (184 and $149 \mathrm{mg}$, respectively, 30\% in total) as a greenishyellow oil.

28 ${ }^{\mathrm{a}}$. TLC (20\% EtOAc in hexanes): $\mathrm{R}_{\mathrm{f}}=0.36 ;[\alpha]_{20}{ }^{\mathrm{D}}+4.4\left(\right.$ c $\left.1.0, \mathrm{CHCl}_{3}\right) ;{ }^{1} \mathrm{H}$ NMR: $\delta=7.62-$ $7.55(\mathrm{~m}, 4 \mathrm{H}), 7.46-7.34(\mathrm{~m}, 8 \mathrm{H}), 6.41$ (br s, 1H), 5.24 (s, 1H), 3.61-3.49 (m, 2H), 1.83 (d, J=4.9 $\mathrm{Hz}, 1 \mathrm{H}), 1.79-1.67(\mathrm{~m}, 1 \mathrm{H}), 1.66-1.54(\mathrm{~m}, 2 \mathrm{H}), 1.53(\mathrm{~s}, 9 \mathrm{H}), 1.38(\mathrm{~d}, J=4.9 \mathrm{~Hz}, 1 \mathrm{H}), 1.35-1.24$ $(\mathrm{m}, 1 \mathrm{H}), 1.00(\mathrm{~s}, 9 \mathrm{H}) ;{ }^{13} \mathrm{C} \mathrm{NMR}: \delta=171.3,164.5,144.3,141.2,135.5,133.6,129.7,127.6$, $122.0,107.9,82.9,75.8,63.0,40.8,36.4,29.9,28.1,26.7,24.1,22.6,19.1$; IR (film): $v_{\max }=$ 1784, 1727, $1506 \mathrm{~cm}^{-1}$; HRMS (ESI+): $\mathrm{m} / \mathrm{z}$ calcd. for $\mathrm{C}_{33} \mathrm{H}_{40} \mathrm{O}_{6} \mathrm{NaSi} 583.2492\left(\mathrm{M}+\mathrm{Na}^{+}\right)$; found: 583.2474 .

28b. TLC (20\% EtOAc in hexanes): $\mathrm{R}_{\mathrm{f}}=0.43 ;[\alpha]_{20}{ }^{\mathrm{D}}-27\left(\right.$ c $\left.1.0, \mathrm{CHCl}_{3}\right) ;{ }^{1} \mathrm{H}$ NMR: $\delta=7.70-$ $7.60(\mathrm{~m}, 4 \mathrm{H}), 7.47-7.32(\mathrm{~m}, 8 \mathrm{H}), 6.38($ br s, $1 \mathrm{H}), 5.41(\mathrm{~s}, 1 \mathrm{H}), 3.72-3.55(\mathrm{~m}, 2 \mathrm{H}), 2.06(\mathrm{dd}, J=$ $11.7,10.4 \mathrm{~Hz}, 1 \mathrm{H}), 1.86(\mathrm{~d}, J=4.9 \mathrm{~Hz}, 1 \mathrm{H}), 1.77-1.67(\mathrm{~m}, 2 \mathrm{H}), 1.55-1.45(\mathrm{~m}, 2 \mathrm{H}), 1.49(\mathrm{~s}, 9 \mathrm{H})$, 1.04 (s, 9H); ${ }^{13} \mathrm{C}$ NMR: $\delta=170.7,164.6,143.9,140.4,135.5,133.6,129.7,127.7,120.9,108.6$, 82.9, 74.5, 63.2, 42.5, 36.0, 29.5, 28.0, 26.8, 25.0, 22.8, 19.2; IR (film): $v_{\max }=1787,1721,1500$ $\mathrm{cm}^{-1}$; HRMS (ESI+): $\mathrm{m} / \mathrm{z}$ calcd. for C33H40O6NaSi $583.2493\left(\mathrm{M}+\mathrm{Na}^{+}\right)$; found: 583.2482 .

(4S)-Furan-3-yl-(5S)-(3-hydroxypropyl)-2-oxo-3-oxabicyclo[3.1.0]hexane-(1S)-carboxylic acid tert-butyl ester (29a). To a stirred solution of cyclopropanolactone 28a (0.38 g, $0.67 \mathrm{mmol}, 100 \mathrm{~mol} \%)$ in THF $(1.4 \mathrm{~mL})$ at $0{ }^{\circ} \mathrm{C}$ was added TBAF $(1 \mathrm{M}$ in THF, $1.34 \mathrm{~mL}$, $1.3 \mathrm{mmol}, 200 \mathrm{~mol} \%$ ) dropwise. After $30 \mathrm{~min}$ at $0{ }^{\circ} \mathrm{C}$, the brown solution was allowed to warm to $\mathrm{rt}$ and after $2 \mathrm{~h}$, sat. aq. $\mathrm{NH}_{4} \mathrm{Cl}(2 \mathrm{~mL})$ was added. The aqueous layer was extracted with EtOAc $(3 \times 5 \mathrm{~mL})$ and the organic extracts were washed with brine, dried, filtered and concentrated. Flash column chromatography (silica, 0-2\% $\mathrm{MeOH}$ in $\mathrm{CH}_{2} \mathrm{Cl}_{2}$ with $2 \% \mathrm{Et}_{3} \mathrm{~N}$ ) provided alcohol 29a as a brown oil $(0.123 \mathrm{~g}, 57 \%)$. TLC (50\% EtOAc in hexanes): $\mathrm{R}_{\mathrm{f}}=0.18$;

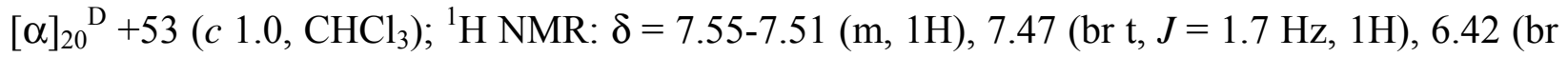
dd, $J=1.7,0.7 \mathrm{~Hz}, 1 \mathrm{H}), 5.33(\mathrm{~s}, 1 \mathrm{H}), 3.55$ (br t, $J=5.9 \mathrm{~Hz}, 2 \mathrm{H}), 1.87$ (d, $J=4.9 \mathrm{~Hz}, 1 \mathrm{H}), 1.72-$ $1.55(\mathrm{~m}, 3 \mathrm{H}), 1.53(\mathrm{~s}, 9 \mathrm{H}), 1.43(\mathrm{dd}, J=4.9,1.5 \mathrm{~Hz}, 1 \mathrm{H}), 1.35-1.28(\mathrm{~m}, 1 \mathrm{H}) ;{ }^{13} \mathrm{C} \mathrm{NMR:} \delta=$ $171.2,164.5,144.4,141.3,122.0,107.9,83.1,75.8,62.0,40.9,36.3,29.9,28.1,23.8,22.8$; IR 
(film): $v_{\max }=3523,1778,1725,1507 \mathrm{~cm}^{-1}$; HRMS (ESI+): $\mathrm{m} / \mathrm{z}$ calcd. for $\mathrm{C}_{17} \mathrm{H}_{22} \mathrm{O}_{6} \mathrm{Na} 345.1314$ $\left(\mathrm{M}+\mathrm{Na}^{+}\right)$; found: 345.1302 .

(4S)-Furan-3-yl-(5R)-(3-hydroxypropyl)-2-oxo-3-oxabicyclo[3.1.0]hexane-(1R)-carboxylic acid tert-butyl ester (29b): Prepared from 28a as described for 29a. Reaction time $3 \mathrm{~h}$. 80\% yield. TLC (50\% EtOAc in hexanes): $\mathrm{R}_{\mathrm{f}}=0.22 ;[\alpha]_{20}{ }^{\mathrm{D}}-74\left(c 1.0, \mathrm{CHCl}_{3}\right) ;{ }^{1} \mathrm{H} \mathrm{NMR}: \delta=7.50-$ $7.48(\mathrm{~m}, 1 \mathrm{H}), 7.44$ (t, $J=1.8 \mathrm{~Hz}, 1 \mathrm{H}), 6.41$ (br dd, $J=1.8,0.7 \mathrm{~Hz}, 1 \mathrm{H}), 5.48(\mathrm{~s}, 1 \mathrm{H}), 3.70-3.57$ (m, 2H), $2.04(\mathrm{td}, J=11.2,1.2 \mathrm{~Hz}, 1 \mathrm{H}), 1.88(\mathrm{~d}, J=5.1 \mathrm{~Hz}, 1 \mathrm{H}), 1.82-1.54(\mathrm{~m}, 3 \mathrm{H}), 1.52(\mathrm{~s}$, 9H), 1.53-1.51 (m, 1H); ${ }^{13} \mathrm{C}$ NMR: $\delta=170.6,164.7,144.0,140.5,120.8,108.7,83.1,74.4,62.1$, 42.4, 36.0, 29.4, 28.0, 24.8, 23.0; IR (film): $v_{\max }=3413,1781,1719,1505 \mathrm{~cm}^{-1}$; HRMS (ESI+): $\mathrm{m} / \mathrm{z}$ calcd. for $\mathrm{C}_{17} \mathrm{H}_{22} \mathrm{O}_{6} \mathrm{Na} 345.1314\left(\mathrm{M}+\mathrm{Na}^{+}\right)$; found: 345.1306 .

(4S)-Furan-3-yl-2-oxo-(5S)-(3-phenylsulfanylpropyl)-3-oxabicyclo[3.1.0]hexane-(1S)carboxylic acid tert-butyl ester (30a). A stirred solution of alcohol 29a $(0.12 \mathrm{~g}, 0.38 \mathrm{mmol}$, $100 \mathrm{~mol} \%)$ and $(\mathrm{SPh})_{2}(0.13 \mathrm{~g}, 0.45 \mathrm{mmol}, 120 \mathrm{~mol} \%)$ in pyridine $(1.9 \mathrm{~mL})$ at $0{ }^{\circ} \mathrm{C}$ was bubbled with argon for $10 \mathrm{~min}$ after which freshly distilled $\mathrm{Bu}_{3} \mathrm{P}(0.19 \mathrm{~mL}, 0.76 \mathrm{mmol}, 200 \mathrm{~mol} \%)$ was added. After $15 \mathrm{~min}$ at $0{ }^{\circ} \mathrm{C}$, the solution was allowed to warm to rt. After $1.5 \mathrm{~h}, \mathrm{Et}_{2} \mathrm{O}(10 \mathrm{~mL})$ was added and the solution was washed with sat. aq. $\mathrm{NaHCO}_{3}(5 \mathrm{~mL})$ and brine, dried, filtered and concentrated. Flash column chromatography (silica, 10-15\% EtOAc in hexanes) provided sulfide 30a as a colorless oil $(0.118 \mathrm{~g}, 75 \%)$. TLC (50\% EtOAc in hexanes): $\mathrm{R}_{\mathrm{f}}=0.65 ;[\alpha]_{20}{ }^{\mathrm{D}}$ $+41\left(\right.$ c 1.0, $\left.\mathrm{CHCl}_{3}\right) ;{ }^{1} \mathrm{H} \mathrm{NMR}: \delta=7.47$ (br t, $\left.J=1.6 \mathrm{~Hz}, 1 \mathrm{H}\right), 7.36(\mathrm{~s}, 1 \mathrm{H}), 7.33-7.17(\mathrm{~m}, 5 \mathrm{H})$, 6.39-6.35 (m, 1H), $5.23(\mathrm{~s}, 1 \mathrm{H}), 2.88-2.71(\mathrm{~m}, 2 \mathrm{H}), 1.84(\mathrm{~d}, J=5.0 \mathrm{~Hz}, 1 \mathrm{H}), 1.76-1.60(\mathrm{~m}, 3 \mathrm{H})$, $1.53(\mathrm{~s}, 9 \mathrm{H}), 1.40(\mathrm{dd}, J=5.0,1.2 \mathrm{~Hz}, 1 \mathrm{H}), 1.37-1.23(\mathrm{~m}, 1 \mathrm{H}) ;{ }^{13} \mathrm{C} \mathrm{NMR}: \delta=171.0,164.4$, $144.4,141.2$, 135.7, 129.4, 128.9, 126.2, 121.8, 107.7, 83.1, 75.6, 40.7, 36.1, 33.2, 28.1, 26.54, 26.48, 22.7; IR (film): $v_{\max }=1783,1725,1584,1506 \mathrm{~cm}^{-1}$; HRMS (ESI+): $\mathrm{m} / \mathrm{z}$ calcd. for $\mathrm{C}_{23} \mathrm{H}_{26} \mathrm{O}_{5} \mathrm{NaS} 437.1399\left(\mathrm{M}+\mathrm{Na}^{+}\right)$; found: 437.1396.

(4S)-Furan-3-yl-2-oxo-(5R)-(3-phenylsulfanylpropyl)-3-oxabicyclo[3.1.0]hexane-(1R)carboxylic acid tert-butyl ester (30b). Prepared from 29a as described for 30a. Reaction time 2.5 h. $65 \%$ yield. TLC (50\% EtOAc in hexanes): $\mathrm{R}_{\mathrm{f}}=0.70 ;[\alpha]_{20}{ }^{\mathrm{D}}-49\left(\mathrm{c} 1.0, \mathrm{CHCl}_{3}\right) ;{ }^{1} \mathrm{H} \mathrm{NMR}$ : $\delta=7.43($ br t, $J=1.7 \mathrm{~Hz}, 1 \mathrm{H}), 7.42-7.40(\mathrm{~m}, 1 \mathrm{H}), 7.33-7.25(\mathrm{~m}, 4 \mathrm{H}), 7.23-7.16(\mathrm{~m}, 1 \mathrm{H}), 6.35$ (br $\mathrm{dd}, J=1.7,0.8 \mathrm{~Hz}, 1 \mathrm{H}), 5.36(\mathrm{~s}, 1 \mathrm{H}), 2.96-2.78(\mathrm{~m}, 2 \mathrm{H}), 2.07$ (td, $J=11.9,1.8 \mathrm{~Hz}, 1 \mathrm{H}), 1.86$ (d, $J=5.1 \mathrm{~Hz}, 1 \mathrm{H}), 1.83-1.73(\mathrm{~m}, 2 \mathrm{H}), 1.70-1.60(\mathrm{~m}, 1 \mathrm{H}), 1.49(\mathrm{~s}, 9 \mathrm{H}), 1.51-1.45(\mathrm{~m}, 1 \mathrm{H}) ;{ }^{13} \mathrm{C}$ NMR: $\delta=170.3,164.5,143.9,140.5,135.6,129.7,129.0,126.4,108.6,99.8,83.1,74.3,42.1$, 36.0, 33.7, 28.0, 27.4, 26.0, 22.7; IR (film): $v_{\max }=1785,1719,1584,1504 \mathrm{~cm}^{-1}$; HRMS (ESI + ): $\mathrm{m} / \mathrm{z}$ calcd. for $\mathrm{C}_{23} \mathrm{H}_{26} \mathrm{O}_{5} \mathrm{NaS} 437.1399\left(\mathrm{M}+\mathrm{Na}^{+}\right)$; found: 437.1388 .

(5S)-(3-Benzenesulfonylpropyl)-(4S)-furan-3-yl-2-oxo-3-oxabicyclo[3.1.0]hexane-(1S)carboxylic acid tert-butyl ester (31a). To a stirred solution of sulfide 30a (82 $\mathrm{mg}, 0.20 \mathrm{mmol}$, $100 \mathrm{~mol} \%)$ in $\mathrm{CH}_{2} \mathrm{Cl}_{2}(1.0 \mathrm{~mL})$ at $0{ }^{\circ} \mathrm{C}$ was added $\mathrm{NaHCO}_{3}(0.17 \mathrm{~g}, 2.0 \mathrm{mmol}, 1000 \mathrm{~mol} \%)$ and m-CPBA (70\%, $0.11 \mathrm{~g}, 0.46 \mathrm{mmol}, 230 \mathrm{~mol} \%)$. After $60 \mathrm{~min}$, sat. aq. $\mathrm{NaHCO}_{3}(2 \mathrm{~mL})$ was added and the aqueous phase was extracted with $\mathrm{CH}_{2} \mathrm{Cl}_{2}(3 \times 5 \mathrm{~mL})$. The organic extracts were washed with brine, dried, filtered and concentrated. Flash column chromatography (silica, 33$50 \%$ EtOAc in hexanes) provided sulfone 31a as a white foam (77 mg, 88\%). TLC (50\% EtOAc 
in hexanes): $\mathrm{R}_{\mathrm{f}}=0.43 ;[\alpha]_{20}{ }^{\mathrm{D}}+29\left(\mathrm{c} 1.0, \mathrm{CHCl}_{3}\right) ;{ }^{1} \mathrm{H}$ NMR: $\delta=7.87-7.81(\mathrm{~m}, 2 \mathrm{H}), 7.71-7.69$ (m, 1H), 7.61-7.54 (m, 2H), 7.45 (br t, $J=1.6 \mathrm{~Hz}, 1 \mathrm{H}), 7.39$ (br s, 1H), 6.33 (br dd, $J=1.6,0.7$ $\mathrm{Hz}, 1 \mathrm{H}), 5.25(\mathrm{~s}, 1 \mathrm{H}), 3.07-2.85(\mathrm{~m}, 2 \mathrm{H}), 1.81(\mathrm{~d}, J=5.1 \mathrm{~Hz}, 1 \mathrm{H}), 1.83-1.62(\mathrm{~m}, 3 \mathrm{H}), 1.49$ (s, 9H), $1.42(\mathrm{dd}, J=5.1,1.3 \mathrm{~Hz}, 1 \mathrm{H}), 1.31-1.21(\mathrm{~m}, 1 \mathrm{H}) ;{ }^{13} \mathrm{C} \mathrm{NMR}: \delta=170.6,164.2,144.6,141.2$, $138.9,133.9,129.4,127.9,121.6,107.6,83.4,75.3,55.5,40.3,35.9,28.1,26.2,22.6,20.3$; IR (film): $v_{\max }=1781,1724,1586,1506 \mathrm{~cm}^{-1}$; HRMS (ESI+): $\mathrm{m} / \mathrm{z}$ calcd. for $\mathrm{C}_{23} \mathrm{H}_{26} \mathrm{O}_{7} \mathrm{NaS}$ 469.1297 $\left(\mathrm{M}+\mathrm{Na}^{+}\right)$; found: 469.1289 .

(5R)-(3-Benzenesulfonylpropyl)-(4S)-furan-3-yl-2-oxo-3-oxabicyclo[3.1.0]hexane-(1R)carboxylic acid tert-butyl ester (31b). Prepared from 30a as described for 31a. Reaction time 2 h. $80 \%$ yield. TLC (50\% EtOAc in hexanes): $\mathrm{R}_{\mathrm{f}}=0.53 ;[\alpha]_{20}{ }^{\mathrm{D}}-54\left(c 1.0, \mathrm{CHCl}_{3}\right) ;{ }^{1} \mathrm{H} \mathrm{NMR}: \delta$ $=7.89-7.83(\mathrm{~m}, 2 \mathrm{H}), 7.70-7.64(\mathrm{~m}, 1 \mathrm{H}), 7.62-7.53(\mathrm{~m}, 2 \mathrm{H}), 7.48-7.42(\mathrm{~m}, 2 \mathrm{H}), 6.41-6.37(\mathrm{~m}$, $1 \mathrm{H}), 5.40(\mathrm{~s}, 1 \mathrm{H}), 3.02(\mathrm{t}, J=7.7 \mathrm{~Hz}, 2 \mathrm{H}), 2.09-1.90(\mathrm{~m}, 2 \mathrm{H}), 1.83(\mathrm{~d}, J=5.2 \mathrm{~Hz}, 1 \mathrm{H}), 1.85-1.63$ $(\mathrm{m}, 2 \mathrm{H}), 1.52(\mathrm{~d}, J=5.2 \mathrm{~Hz}, 1 \mathrm{H}), 1.45(\mathrm{~s}, 9 \mathrm{H}) ;{ }^{13} \mathrm{C} \mathrm{NMR}: \delta=170.0,164.5,144.1,140.7,138.9$, 133.9, 129.4, 127.9, 120.5, 108.6, 83.4, 74.1, 55.7, 41.7, 35.8, 27.9, 27.1, 22.7, 20.0; IR (film): $v_{\max }=1784,1718,1586,1505 \mathrm{~cm}^{-1}$; HRMS (ESI+): $\mathrm{m} / \mathrm{z}$ calcd. for $\mathrm{C}_{23} \mathrm{H}_{26} \mathrm{O}_{7} \mathrm{NaS} 469.1297(\mathrm{M}+$ $\mathrm{Na}^{+}$); found: 469.1311 .

Ketosulfone 32a. To a stirred solution of sulfone 31a (77 mg, $0.17 \mathrm{mmol}, 100 \mathrm{~mol} \%)$ in THF (3.5 mL, freshly distilled from $\left.\mathrm{LiAlH}_{4}\right)$ at $-100{ }^{\circ} \mathrm{C}$ was added $\mathrm{n}-\mathrm{BuLi}(0.16 \mathrm{~mL}, 0.35 \mathrm{mmol}, 200$ mol\%) dropwise. After $15 \mathrm{~min}$, more n-BuLi (15 $\mu \mathrm{L}, 34 \mu \mathrm{mol}, 20 \mathrm{~mol} \%$ ) was added because some starting material was evident by TLC analysis. After 25 min, sat. aq. $\mathrm{NH}_{4} \mathrm{Cl}$ was added and the mixture was allowed to warm to rt. The aqueous phase was extracted with EtOAc and the organic extracts were washed with brine, dried, filtered and concentrated. Flash column chromatography (silica, 40-60\% EtOAc in hex) provided ketosulfone 32a as mixture of diastereomers $(\mathbf{a}: \mathbf{b}=1: 0.8,33 \mathrm{mg}, 55 \%)$. TLC (50\% EtOAc in hexanes): $\mathrm{R}_{\mathrm{f}}=0.1 ;{ }^{1} \mathrm{H}$ NMR: $\delta=$ 7.87-7.83 (m, 2H, b), 7.82-7.77 (m, 2H, a), 7.72-7.66 (m, 2H, a+b), 7.60-7.54 (m, 5H, 3a+2b), 7.48 (br t, $J=1.7 \mathrm{~Hz}, 1 \mathrm{H}, \mathrm{a}), 7.46-7.43$ (m, 2H, b), 6.73 (br dd, $J=1.7,0.7 \mathrm{~Hz}, 1 \mathrm{H}, \mathrm{a}), 6.20$ (br t, $J=1.4 \mathrm{~Hz}, 1 \mathrm{H}, \mathrm{b}), 5.47$ (s, 1H, a), 5.45 (s, 1H, b), 3.76 (dd, $J=10.0,8.7 \mathrm{~Hz}, 1 \mathrm{H}, \mathrm{b}), 3.59$ (dd, $J$ $=5.6,1.9 \mathrm{~Hz}, 1 \mathrm{H}, \mathrm{a}), 2.88-2.80(\mathrm{~m}, 1 \mathrm{H}, \mathrm{a}), 2.86(\mathrm{~d}, J=6.4 \mathrm{~Hz}, 1 \mathrm{H}, \mathrm{b}), 2.48-2.30(\mathrm{~m}, 3 \mathrm{H}, \mathrm{a}+2 \mathrm{~b})$, 2.20 (dt, $J=14.2,4.1 \mathrm{~Hz}, 1 \mathrm{H}, \mathrm{b}), 2.02-1.89$ (m, 2H, a), 1.92 (d, $J=5.9 \mathrm{~Hz}, 1 \mathrm{H}, \mathrm{a}), 1.81-1.71$ (m, 1H, b), 1.81 (d, $J=6.4 \mathrm{~Hz}, 1 \mathrm{H}, \mathrm{b}), 1.70$ (d, $J=5.9 \mathrm{~Hz}, 1 \mathrm{H}, \mathrm{a}) ;{ }^{13} \mathrm{C}$ NMR: $\delta=189.7$ (a), 189.2 (b), 168.0 (a/b), 167.9 (a/b), 144.7 (b), 144.2 (a), 141.7 (a), 140.6 (b), 138.2 (a), 137.6 (b), 134.39 (a), 134.35 (b), 129.23 (a/b), 129.17 (2a/b), 129.1 (a/b), 121.5 (b), 120.7 (a), 109.2 (a), 107.8 (b), 77.2 (a), 75.7 (b), 68.5 (b), 67.2 (a), 40.3 (b), 35.6 (b), 35.0 (a), 34.8 (a), 23.6 (b), 23.2 (a), 20.0 (b), 19.9 (b), 19.3 (a), 18.3 (a); IR (film): $v_{\max }=1780,1704,1583,1503 \mathrm{~cm}^{-1}$; HRMS (ESI+) $\mathrm{m} / \mathrm{z}$ calcd. for $\mathrm{C}_{19} \mathrm{H}_{16} \mathrm{O}_{6} \mathrm{NaS} 395.0565\left(\mathrm{M}+\mathrm{Na}^{+}\right)$; found: 395.0572 .

Methyl ketone 33. To a stirred solution of ketosulfone 32a (7.0 mg, $19 \mu \mathrm{mol}, 100 \mathrm{~mol} \%)$ in THF: $\mathrm{H}_{2} \mathrm{O}(9: 1,1.0 \mathrm{~mL})$ at $0{ }^{\circ} \mathrm{C}$ was added commercial aluminum foil $(1.5 \mathrm{mg}, 57 \mu \mathrm{mol}$, $300 \mathrm{~mol} \%$ ), which had been immersed in $\mathrm{HgCl}_{2}$ (aq., $2 \%$ ), washed with $\mathrm{EtOH}$ and $\mathrm{Et}_{2} \mathrm{O}$ and cut into small pieces. After $1 \mathrm{~h}$, more Al-foil $(1.5 \mathrm{mg}, 57 \mu \mathrm{mol}, 300 \mathrm{~mol} \%)$ was added and after $4 \mathrm{~h}$, the mixture was filtered through a pad of Celite ${ }^{\circledR}$, dried, filtered and concentrated. Flash column 
chromatography (silica, 33-50\% EtOAc in hex) provided methyl ketone 33 (2 $\mathrm{mg}, 45 \%)$. TLC (50\% EtOAc in hexanes): $\mathrm{R}_{\mathrm{f}}=0.5 ;[\alpha]_{20}{ }^{\mathrm{D}}-33$ (c $0.23, \mathrm{CHCl}_{3} ; 46 \%$ ee); ${ }^{1} \mathrm{H}$ NMR: $\delta=7.51$ (s, $1 \mathrm{H}), 7.48(\mathrm{~s}, 1 \mathrm{H}), 6.40(\mathrm{~s}, 1 \mathrm{H}), 5.08(\mathrm{~s}, 1 \mathrm{H}), 3.30(\mathrm{br} \mathrm{s}, 1 \mathrm{H}), 2.48-2.33(\mathrm{~m}, 2 \mathrm{H}), 2.06-1.97(\mathrm{~m}$, $1 \mathrm{H}), 1.78-1.66(\mathrm{~m}, 2 \mathrm{H}), 1.33-1.20(\mathrm{~m}, 1 \mathrm{H}), 1.29(\mathrm{~s}, 3 \mathrm{H}) ;{ }^{13} \mathrm{C}$ NMR: $\delta=202.7,170.8,143.8$, 140.2, 118.3, 108.6, 82.9, 63.1, 49.6, 39.6, 28.3, 21.3, 20.6; IR (film): $v_{\max }=1776,1716,1504$ $\mathrm{cm}^{-1}$; HRMS (ESI+): $\mathrm{m} / \mathrm{z}$ calcd. for $\mathrm{C}_{13} \mathrm{H}_{14} \mathrm{O}_{4} \mathrm{Na} 257.0790\left(\mathrm{M}+\mathrm{Na}^{+}\right)$; found: 257.0779 .

Ketosulfone 32b. To a solution of sulfone 31b $(0.122 \mathrm{~g}, 0.273 \mathrm{mmol}, 100 \mathrm{~mol} \%)$ in THF $\left(2.7 \mathrm{~mL}\right.$, freshly distilled from $\left.\mathrm{LiAlH}_{4}\right)$ at $-100{ }^{\circ} \mathrm{C}$ was added KHMDS ( $1 \mathrm{M}$ in toluene, $1.4 \mathrm{~mL}$, $0.68 \mathrm{mmol}, 250 \mathrm{~mol} \%$ ) dropwise over $5 \mathrm{~min}$. After $1.5 \mathrm{~h}$, sat. aq. $\mathrm{NH}_{4} \mathrm{Cl}$ was added and the mixture was allowed to warm to rt. The aqueous phase was extracted with EtOAc and the organic extracts were washed with brine, dried, filtered and concentrated. Flash column chromatography (silica, 33-50\% EtOAc in hex) provided sulfone 31b $(28 \mathrm{mg}$ ) and ketosulfone 32b as mixture of diastereomers (a:b $=1: 0.4,28 \mathrm{mg}, 36 \%$ based on recovered 31b). TLC (50\% EtOAc in hexanes): $\mathrm{R}_{\mathrm{f}}=0.2 ;{ }^{1} \mathrm{H}$ NMR: $\delta=7.89-7.84(\mathrm{~m}, 2 \mathrm{H}, \mathrm{b}), 7.80-7.75(\mathrm{~m}, 2 \mathrm{H}, \mathrm{a}), 7.73-7.64$ $(\mathrm{m}, 2 \mathrm{H}, \mathrm{a}+\mathrm{b}), 7.62-7.53(\mathrm{~m}, 4 \mathrm{H}, 2 \mathrm{a}+2 \mathrm{~b}), 7.53-7.50(\mathrm{~m}, 1 \mathrm{H}, \mathrm{a}), 7.50-7.46(\mathrm{~m}, 3 \mathrm{H}, \mathrm{a}+2 \mathrm{~b}), 6.44-$ $6.41(\mathrm{~m}, 1 \mathrm{H}, \mathrm{a}), 6.40-6-38(\mathrm{~m}, 1 \mathrm{H}, \mathrm{b}), 5.59$ (s, 1H, a), 5.30 (s, 1H, b), 3.93 (dd, J = 10.4, 7.4 Hz, $1 \mathrm{H}, \mathrm{b}), 3.60$ (dd, $J=5.0,2.1 \mathrm{~Hz}, 1 \mathrm{H}, \mathrm{a}), 2.93$ (ddt, $J=15.6,5.6,2.1 \mathrm{~Hz}, 1 \mathrm{H}, \mathrm{a}), 2.74(\operatorname{td}, J=$ 13.9, $5.6 \mathrm{~Hz}, 1 \mathrm{H}, \mathrm{a}), 2.66$, (d, $J=6.5 \mathrm{~Hz}, 1 \mathrm{H}, \mathrm{b}), 2.56-2.41$ (m, 3H, b), 2.34 (ddd, $J=13.9,5.0$, $2.1 \mathrm{~Hz}, 1 \mathrm{H}, \mathrm{a}), 2.24-2.12(\mathrm{~m}, 1 \mathrm{H}, \mathrm{b}), 1.97$ (ddt, $J=15.6,13.9,5.0 \mathrm{~Hz}, 1 \mathrm{H}, \mathrm{a}), 1.73$ (d, $J=6.5 \mathrm{~Hz}$, 1H, b), 1.67 (d, $J=6.2 \mathrm{~Hz}, 1 \mathrm{H}, \mathrm{a}), 1.63(\mathrm{~d}, J=6.2 \mathrm{~Hz}, 1 \mathrm{H}, \mathrm{a}) ;{ }^{13} \mathrm{C}$ NMR: $\delta=189.8(\mathrm{a}+\mathrm{b}), 168.2$ (a), 168.0 (b), 144.3 (b), 144.2 (a), 140.1 (b), 140.0 (a), 137.4 (a+b), 134.6 (a), 134.3 (b), 129.4 (a), 129.3 (b), 129.2 (b), 129.1 (a), 120.8 (a), 120.5 (b), 108.3 (a), 108.2 (b), 77.3 (a), 76.2 (b), 68.5 (b), 68.3 (a), 39.6 (b), 36.9 (b), 35.4 (a), 33.5 (a), 21.8 (b), 21.1 (b), 20.5 (a+b), 20.0 (a), 19.4 (a); IR (film): $v_{\max }=1784,1708,1635,1503 \mathrm{~cm}^{-1}$; HRMS (ESI+): $\mathrm{m} / z$ calcd. for $\mathrm{C}_{19} \mathrm{H}_{16} \mathrm{O}_{6} \mathrm{NaS} 395.0565\left(\mathrm{M}+\mathrm{Na}^{+}\right)$; found: 395.0532 .

Cyclopropyl ketone 34. To a stirred solution of ketosulfone 32b (10.0 mg, $27 \mu \mathrm{mol}, 100 \mathrm{~mol} \%$ ) in THF: $\mathrm{H}_{2} \mathrm{O}(9: 1,1.4 \mathrm{~mL})$ at $0{ }^{\circ} \mathrm{C}$ was added commercial aluminum foil $(3.6 \mathrm{mg}, 0.13 \mathrm{mmol}$, $500 \mathrm{~mol} \%$ ), which had been immersed in $\mathrm{HgCl}_{2}$ (aq., 2\%), washed with $\mathrm{EtOH}$ and $\mathrm{Et}_{2} \mathrm{O}$ and cut into small pieces. After $30 \mathrm{~min}$, the cooling bath was removed. After $2 \mathrm{~h}$ more Al-foil $(3.6 \mathrm{mg}$, $0.13 \mathrm{mmol}, 500 \mathrm{~mol} \%$ ) was added and after $3 \mathrm{~h}$, the mixture was filtered through a pad of Celite $^{\circledR}$, dried, filtered and concentrated. Flash column chromatography (silica, 33-50\% EtOAc in hex) provided cyclopropyl ketone 34 (2.5 mg, 40\%). TLC (50\% EtOAc in hexanes): $\mathrm{R}_{\mathrm{f}}=0.2$; $[\alpha]_{20}{ }^{\mathrm{D}}-71\left(c 0.2, \mathrm{CHCl}_{3}\right) ;{ }^{1} \mathrm{H}$ NMR: $\delta=7.51-7.48(\mathrm{~m}, 1 \mathrm{H}), 7.46(\mathrm{t}, J=1.6 \mathrm{~Hz}, 1 \mathrm{H}), 6.42-6.40$ (m, 1H), $5.40(\mathrm{~s}, 1 \mathrm{H}), 2.42(\mathrm{dt}, J=18,4.6 \mathrm{~Hz}, 1 \mathrm{H}), 2.33(\mathrm{dt}, J=13,4.6 \mathrm{~Hz}, 1 \mathrm{H}), 2.24(\mathrm{ddd}, J=$ $18,11,6.2 \mathrm{~Hz}, 1 \mathrm{H}), 2.09$ (ddd, $J=13,11,4.9 \mathrm{~Hz}, 1 \mathrm{H}), 2.03-1.92(\mathrm{~m}, 1 \mathrm{H}), 1.94(\mathrm{~d}, J=6.0 \mathrm{~Hz}$, $1 \mathrm{H}), 1.82-1.69(\mathrm{~m}, 1 \mathrm{H}), 1.63(\mathrm{~d}, J=6.0 \mathrm{~Hz}, 1 \mathrm{H}) ;{ }^{13} \mathrm{C}$ NMR: $\delta=197.6,168.9,144.2,139.9$, 121.0, 108.3, 76.9, 37.2, 36.8, 35.4, 23.9, 21.0, 19.3; IR (film): $v_{\max }=1785,1695,1505 \mathrm{~cm}^{-1}$; HRMS (ESI+) $\mathrm{m} / \mathrm{z}$ calcd. for $\mathrm{C}_{13} \mathrm{H}_{12} \mathrm{O}_{4} \mathrm{Na} 255.0633\left(\mathrm{M}+\mathrm{Na}^{+}\right)$; found: 255.0640 . 


\section{Acknowledgements}

We thank Dr. Heikki Hassila for informative discussions regarding the cyclopropanation reactions as well as Ministry of Education in Finland, Finnish Cultural Foundation and Helsinki University of Technology for funding.

\section{References and Notes}

1. Mondon, A.; Epe, B. Progr. Chem. Org. Nat. Prod. 1983, 44, 101.

2. Pihko, A. J.; Koskinen, A. M. P. Tetrahedron 2005, 61, 8769.

3. Mondon, A.; Epe, B.; Callsen, H. Liebigs Ann. Chem. 1983, 1760.

4. Mulholland, D. A.; McFarland, K.; Randrianarivelojosia, M.; Rabarison, H. Phytochemistry 2004, 65, 2929.

5. Mulholland, D.; Mahomed, H.; Kotsos, M.; Randrianarivelojosia, M.; Lavaucf, C.; Massiot, G.; Nuzillard, J.-M. Tetrahedron 1999, 55, 11547.

6. Pihko, A. J.; Lundell, K.; Kanerva, L.; Koskinen, A. M. P. Tetrahedron: Asymmetry 2004, $15,1637$.

7. Koskinen, A. M. P.; Muñoz, L. J. Chem. Soc., Chem. Commun. 1990, 1373.

8. Koskinen, A. M. P.; Muñoz, L. J. Org. Chem. 1993, 58, 879.

9. Koskinen, A. M. P.; Hassila, H. J. Org. Chem. 1993, 58, 4479.

10. (a) Koskinen, A. M. P.; Hassila, H. Acta Chem. Scand. 1996, 50, 323. (b) Pellissier, H. Tetrahedron 2008, 64, 7041.

11. Hassila, H. Ph.D. Thesis, University of Oulu, 1998. Transition metal catalysed intramolecular cyclopropanation.

12. Fraile, J. M.; García, J. I.; Martínez-Merino, V.; Mayoral, J. A.; Salvatella, L. J. Am. Chem. Soc. 2001, 123, 7616.

13. Bühl, M.; Terstegen, F.; Löffler, F.; Meynhardt, B.; Kierse, S.; Müller, M.; Näther, C.; Lüning, U. Eur. J. Org. Chem. 2001, 2151.

14. Rasmussen, T.; Jensen, J. F.; Østergaard, N.; Tanner, D.; Ziegler, T.; Norrby, P.-O. Chem. Eur. J. 2002, 8, 177.

15. Evans, D. A.; Woerpel, K. A.; Hinman, M. H.; Faul, M. M. J. Am. Chem. Soc. 1991, 113, 726.

16. Kubas, G. J. Inorg. Synth. 1979, 19, 90.

17. Doyle, M. P.; Peterson, C. S.; Zhou, G.-L.; Nishiyama, H. Chem. Commun. 1997, 211.

18. Fryzuk, M. D.; Jafarpour, L.; Rettig, S. J. Tetrahedron: Asymmetry 1998, 9, 3191.

19. Neises, B.; Steglich, W. Org. Synth. 1990, Coll. Vol. VII, 92.

20. Koskinen, A. M. P.; Muñoz, L. J. Chem. Soc. Chem. Comm. 1990, 652.

21. Regitz, M.; Hocker, J.; Liedhegener, A. Org. Synth. 1973, Coll. Vol. V, 179. 
22. Wenkert, E.; Guo, M.; Lavilla, R.; Porter, B.; Ramachandran, K.; Sheu, J.-H. J. Org. Chem. 1990, 55, 6203.

23. Schinnerl, M.; Böhm, C.; Seitz, M.; Reiser, O. Tetrahedron: Asymmetry 2003, 14, 765.

24. Konosu, T.; Oida, S. Chem. Pharm. Bull. 1991, 39, 2212.

25. Nagakawa, I.; Hata, T. Tetrahedron Lett. 1975, 1409.

26. Tabuchi, H.; Hamamoto, T.; Miki, S.; Tejima, T.; Ichihara, A. J. Org. Chem. 1994, 59, 4749.

27. Staley, S. W. Regioselectivity in the Reductive Cleavage of Cyclopropane Rings by Dissolving Metals. In Selective Organic Transformations; Thyagarajan, B. S., Ed.; WileyInterscience, 1972; Vol. 2, pp 309.

28. von Baeyer, A. Chem. Ber. 1895, 28, 1586.

29. Norin, T. Acta Chem. Scand. 1965, 19, 1289.

30. Dauben, W. G.; Deviny, E. J. J. Am. Chem. Soc. 1966, 31, 3794.

31. Corey, E. J.; Chaykovsky, M. J. Am. Chem. Soc. 1965, 87, 1345.

32. Jia, Y. X.; Li, X.; Wu, B.; Zhao, X. Z.; Tu, Y. Q. Tetrahedron 2002, 58, 1697.

33. Because the ketone functionality in 32a was apparently activating the cyclopropyl ring too much, the ketone was reduced stereoselectively to provide a 1:1 mixture of $\alpha$ - and $\beta$-synhydroxysulfones $\mathbf{3 5}$ in $90 \%$ yield. However, reduction of the hydroxysulfones $\mathbf{3 5}$ with either Raney nickel or aluminum amalgam resulted in recovery of part of the starting material accompanied with decomposition.

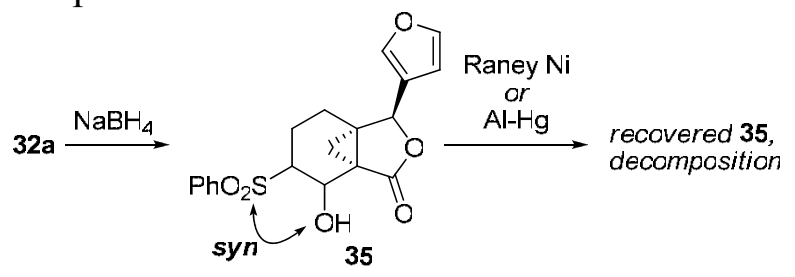

34. Kelly, A. R.; Kerrigan, M. H.; Walsh, P. J. J. Am. Chem. Soc. 2008, 130, 4097.

35. Paquette, L. A.; Hofferberth, J. E. J. Org. Chem. 2003, 68, 2266.

36. Breslow, D. S.; Baumgarten, E.; Hauser, C. R. J. Am. Chem. Soc. 1944, 66, 1286.

37. Gibson, F. S.; Park, M. S.; Rapoport, H. J. Org. Chem. 1994, 59, 7503.

38. Harwood, L. M. Aldrichim. Acta 1985, 18, 25. 